\title{
DS-CDMA with Power Control Error Using Weighted Despreading Sequences over a Multipath Rayleigh Fading Channel
}

\author{
Yuejin Huang, Member, IEEE, and T. S. Ng, Senior Member, IEEE
}

\begin{abstract}
In this paper, closed-form solutions for the average bit error rate (BER) performance of a direct-sequence codedivision multiple-access system with imperfect power control are derived for both coherent and noncoherent receptions operating over a multipath Rayleigh fading channel. The RAKE structure receivers under consideration employ despreading sequences weighted by adjustable exponential chip waveforms optimized for multiple-access interference rejection. The chip-weighting waveforms employed are determined only by one parameter $\gamma$ which leads to easy tuning of the waveforms in practice to achieve the best performance. The results indicate that the number of active users supported at a given BER for the case of $\gamma$ tuned to maximize the average signal to interference plus noise ratio $\hat{\boldsymbol{H}}$ is much larger than the case of $\gamma=0$ (fixed or rectangular despreading sequence). It is shown that imperfect power control affects the irreducible BER for the case of $\gamma=0$. On the other hand, the effect of imperfect power control on BER performance for the case of $\gamma$ tuned to maximize $\hat{H}$ is equivalent to a reduction in the average signal-to-noise ratio, and, hence, system performance can be compensated by increasing the transmitter power. It is further shown that the effect due to imperfect power control on BER performance is significant while that on the maximum value of $\hat{\boldsymbol{H}}$ obtained by tuning $\gamma$ is rather insignificant.
\end{abstract}

Index Terms-Code-division multiple access, RAKE receivers, spread-spectrum communication.

\section{INTRODUCTION}

W ITH THE rapid growth of personal wireless communications, direct-sequence code-division multiple-access (DS-CDMA) technology has attracted considerable attention over the last few years because of its many attractive properties for the wireless medium [1]-[3]. However, as is well known, the major factors which limit the performance, and, hence, the capacity of DS-CDMA systems are multipath fading, multiple-access interference (MAI) and imperfect power control. In many performance analyses of CDMA systems, the assumptions are made that the transmitted power of each user is perfectly controlled and the waveform of despreading sequence in a receiver is the same as the spreading sequence assigned to a reference user [4]-[12].

Manuscript received January 21, 1997; revised June 23, 1997. This work was supported by the Hong Kong Research Grants Council and the CRCG of the University of Hong Kong.

Y. Huang is with the Department of Electrical Engineering, McGill University, Montreal, P.Q., Canada (e-mail: yhuang@wireless.ece.mcgill.ca).

T. S. Ng is with the Department of Electrical and Electronic Engineering, University of Hong Kong, Hong Kong (e-mail: tsng@eee.hku.hk).

Publisher Item Identifier S 0018-9545(99)05722-9.
A receiver is called correlation receiver when the despreading sequence is the same as the spreading sequence. It has optimal performance in the presence of additive white Gaussian noise (AWGN). However, in a DS-CDMA system, there are two kinds of "noise": MAI and AWGN. The MAI is the sum of many independent couser signals and can be modeled as zero-mean-colored Gaussian process. If the signals of cousers are shifted in time by a random amount distributed uniformly between zero and $T_{c}$ where $T_{c}$ is a chip duration, the MAI becomes a statistically stationary random process [13]. It is easy to see that the optimum receiver derived in AWGN performs badly when the MAI dominates over the AWGN because the MAI is not white. When the MAI and AWGN occur simultaneously, an optimum despreading function has been shown to be the solution of a Fredholm integral equation of the second kind [14, eq. (9-67)] and the corresponding receiver is called the integral equation receiver [15]. Assuming that a DS-CDMA system operates over an AWGN channel, the integral equation has been solved in [15] for the case in which the spreading pulses are rectangular and the resulting despreading function consists of $2 N^{2}$ exponential terms with $N(N+3)$ coefficients where $N$ is the processing gain. In practice, it is difficult to tune to the optimum despreading function when $N$ is large.

In addition to MAI rejection, power control is currently considered indispensable for a successful DS-CDMA system. In a real system, the power control error exists irrespective of power control methods being used. The effect of imperfect power control on performance was analyzed in [16] with the maximum diversity order of two. In practice, a typical DSCDMA system over a terrestrial channel may operate with $L_{R}$-branch diversity for better performance. Therefore, we are motivated to analyze the performance of a DS-CDMA system with power control error using any order of diversity to combat multipath fading.

In this paper, we analyze the performance of a DS-CDMA system with power control error over a multipath Rayleigh fading channel for the uplink. With the purpose of MAI rejection, the RAKE structure receivers under consideration employ the despreading sequences weighted by adjustable exponential chip waveforms [17], [18]. The resulting despreading functions are determined only by one parameter and this leads to easy tuning of the despreading sequences in practice to achieve the best performance in multipath environment. Specifically, this paper analyzes and compares 
the BER performance of a DS-CDMA system using both coherent and noncoherent receptions. Here for brevity, the receivers with coherent detection and noncoherent detection are simply called RAKE receiver and RAKE demodulator, respectively. The RAKE receiver is equivalent to a receiver with maximal ratio combiner and the RAKE demodulator is a noncoherent receiver with equal gain combining for DPSK signaling.

The organization of the paper is as follows. In Section II, we describe the system and channel models. The BER performance of a DS-CDMA system with power control error for both coherent reception and noncoherent reception operating over a multipath Rayleigh fading channel is derived under the proposed weighted despreading sequences in Section III. This is followed by numerical results and discussion of the results in Section IV. Finally, in Section V, conclusions are given.

\section{SyStem AND CHANNEL Models}

\section{A. Transmitter Model}

Suppose there are $K$ CDMA users accessing the channel. User $k$ transmits a binary data sequence $b_{k}(t)$ (data bits are differentially encoded for DPSK) and employs a spreading sequence $a_{k}(t)$ to spread each data bit. The spreading and data sequences for the $k$ th user are given by $a_{k}(t)=$ $\sum_{j=-\infty}^{\infty} a_{j}^{(k)} P_{T_{c}}\left(t-j T_{c}\right)$ and $b_{k}(t)=\sum_{j=-\infty}^{\infty} b_{j}^{(k)} P_{T_{b}}(t-$ $\left.j T_{b}\right)$ where $T_{c}$ and $T_{b}$ are the chip and data durations, respectively, and $P_{x}(y)=1$ for $0<y<x$ and zero otherwise. In our study, $b_{j}^{(k)}$ and $a_{j}^{(k)}$ are modeled as independent random variables taking values -1 or +1 with equal probabilities. It is assumed that there are $N$ chips of a spreading sequence in the interval of each data bit $T_{b}$ and the spreading sequence for each user has period equal to $N$. In other words, $T_{b}=N T_{c}$ and $a_{j}^{(k)}=a_{j+N}^{(k)}$ for all $-\infty<j<+\infty$. The transmitted signal for the $k$ th user is

$$
S_{k}(t)=\sqrt{2 P} G_{k} b_{k}(t) a_{k}(t) \cos \left(\omega_{0} t+\theta_{k}\right)
$$

where the transmitted power $P$ and the carrier frequency $\omega_{0}$ are common to all users and the parameter $\theta_{k}$ is the phase of the $k$ th user. The parameter $G_{k}$ represents the power control error for the $k$ th user and is modeled as a random variable uniformly distributed in $\left[1-\varepsilon_{m}, 1+\varepsilon_{m}\right]$ where $\varepsilon_{m}$ represents the maximum value of power control error for all users. Such a distribution for the parameter $G_{k}$ implies a complete lack of knowledge of the power control error and is a least favorable distribution [16].

\section{B. Channel Model}

In our analysis, we consider a frequency selective multipath channel for the uplink. The equivalent complex low-pass representation of the channel for the $k$ th user is given by

$$
h_{k}(t)=\sum_{l=0}^{L_{P}-1} \beta_{k l l} \delta\left(t-\tau_{k l}\right) e^{j \eta_{k l}}
$$

where random variables $\beta_{k l}, \tau_{k l}$, and $\eta_{k l}$ are the $l$ th path gain, delay and phase, respectively, for the $k$ th user. Furthermore, we assume the following: 1) for different users and paths in each link, the random variables $\left\{\beta_{k l}\right\},\left\{\tau_{k l}\right\}$, and $\left\{\eta_{k l}\right\}$ are all statistically independent; 2) the random phases $\left\{\eta_{k l}\right\}$ are uniformly distributed over $[0,2 \pi]$ and the path delays $\left\{\tau_{k l}\right\}$ are uniformly distributed over $\left.\left[0, T_{b}\right] ; 3\right)$ there are $L_{P}$ paths for each user and these different paths are separated in time from each other by more than $2 T_{c}$; and 4) for each user, the path gain $\beta_{k l}$ is a random variable with Rayleigh distribution given by

$$
p\left(\beta_{k l}\right)= \begin{cases}\frac{\beta_{k l}}{\rho_{k l}} \exp \left(-\frac{\beta_{k l}^{2}}{2 \rho_{k l}}\right), & \beta_{k l} \geq 0 \\ 0, & \beta_{k l}<0\end{cases}
$$

where $E\left[\beta_{k l}^{2}\right]=2 \rho_{k l}$; and 5) the fading rate in the channel is slow compared to the bit rate, so that the random parameters associated with the channel do not vary significantly over two consecutive bit intervals.

At the central station, all user signals, after passing through their own particular channel, are added together and mixed with AWGN $n(t)$ with two-side power spectral density $N_{0} / 2$. Therefore, the input signal at the central station $r(t)$ can be represented by

$$
\begin{aligned}
r(t)= & \sqrt{2 P} \sum_{k=1}^{K} \sum_{l=0}^{L_{P}-1} G_{k} \beta_{k l} b_{k}\left(t-\tau_{k l}\right) a_{k}\left(t-\tau_{k l}\right) \\
& \cdot \cos \left(\omega_{0} t+\phi_{k l}\right)+n_{c}(t) \cos \omega_{0} t-n_{s}(t) \sin \omega_{0} t
\end{aligned}
$$

where $\phi_{k l}=\theta_{k}+\eta_{k l}-\omega_{0} \tau_{k l}$ and the terms $n_{c}(t)$ and $n_{s}(t)$ are low-pass equivalent components of the AWGN $n(t)$. The random phases $\left\{\phi_{k l}\right\}$ are uniformly distributed over $[0,2 \pi]$. The random variables $\left\{G_{k}\right\},\left\{\beta_{k l}\right\},\left\{\tau_{k l}\right\}$, and $\left\{\phi_{k l}\right\}$ are independent of each other.

\section{Receiver Model}

For BPSK modulation, the structure of one of the paths of a RAKE receiver using coherent detection is shown in Fig. 1. For MAI rejection, a bank of single-path matched filters, each of which is matched to different paths, have the same impulse response matched to $2 \hat{a}_{k}(t) \cos \left(\omega_{0} t\right) P_{T_{b}}(t)$ where $\hat{a}_{k}(t)$ is the weighted despreading sequence with details given below. The outputs of all single-path matched filters $\zeta_{k l}(\kappa), l \in\left[0, L_{R}-1\right]$ where $L_{R}$ is the order of diversity, are weighted by the corresponding path gains and then summed to form a single decision variable $\zeta_{k}(\kappa)$. The weighted despreading sequence of the user $k$ 's RAKE receiver can be expressed as [17], [18]

$$
\hat{a}_{k}(t)=\sum_{j=-\infty}^{\infty} a_{j}^{(k)} w_{j}^{(k)}\left(t-j T_{c} \mid\left\{c_{j}^{(k)}, c_{j+1}^{(k)}\right\}\right)
$$

where $c_{j}^{(k)}=a_{j-1}^{(k)} a_{j}^{(k)}$ and $w_{j}^{(k)}\left(t \mid\left\{c_{j}^{(k)}, c_{j+1}^{(k)}\right\}\right)$, for $0 \leq t \leq$ $T_{c}$, is the $j$ th chip-weighting waveform for the $k$ th receiver conditioned on the status of three consecutive chips $\left\{c_{j}^{(k)}\right.$, $\left.c_{j+1}^{(k)}\right\}=\left\{a_{j-1}^{(k)} a_{j}^{(k)}, a_{j}^{(k)} a_{j+1}^{(k)}\right\}$. Note that in [15] the optimum despreading function emphasizes the transitions of the received 


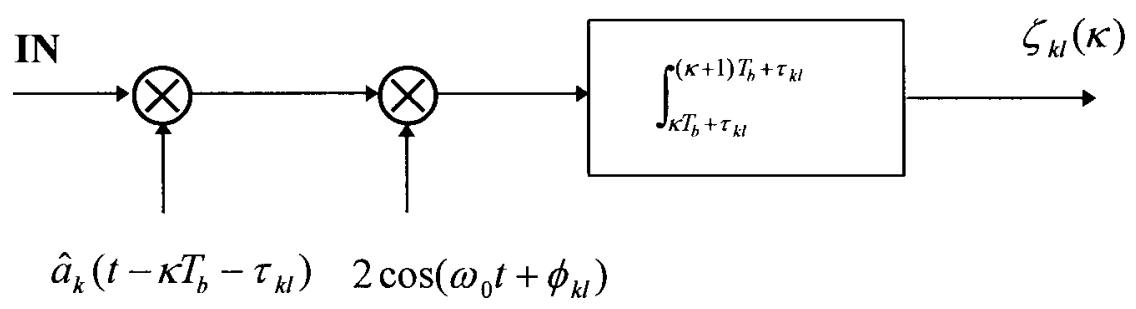

Fig. 1. The structure of path $l$ 's matched filter for the $k$ th user.

signal of the reference user. Therefore, we define

$$
\begin{aligned}
& w_{j}^{(k)}\left(t \mid\left\{c_{j}^{(k)}, c_{j+1}^{(k)}\right\}\right) \\
& = \begin{cases}c w_{1}(t) & \text { if } c_{j}^{(k)}=+1 \text { and } c_{j+1}^{(k)}=+1 \\
c w_{2}(t) & \text { if } c_{j}^{(k)}=-1 \text { and } c_{j+1}^{(k)}=-1 \\
c w_{3}(t) & \text { if } c_{j}^{(k)}=-1 \text { and } c_{j+1}^{(k)}=+1 \\
c w_{4}(t) & \text { if } c_{j}^{(k)}=+1 \text { and } c_{j+1}^{(k)}=-1\end{cases}
\end{aligned}
$$

with the elements of the chip-weighting waveform vector $\left\{c w_{1}(t), c w_{2}(t), c w_{3}(t), c w_{4}(t)\right\}$ given by the following:

$$
\begin{aligned}
& c w_{1}(t)=e^{-\gamma / 2} P_{T_{c}}(t) \\
& c w_{2}(t)=e^{-\gamma t / T_{c}} P_{T_{c} / 2}(t)+e^{-\gamma\left(1-t / T_{c}\right)} P_{T_{c} / 2}\left(t-T_{c} / 2\right) \\
& c w_{3}(t)=e^{-\gamma t / T_{c}} P_{T_{c} / 2}(t)+e^{-\gamma / 2} P_{T_{c} / 2}\left(t-T_{c} / 2\right) \\
& c w_{4}(t)=e^{-\gamma / 2} P_{T_{c} / 2}(t)+e^{-\gamma\left(1-t / T_{c}\right)} P_{T_{c} / 2}\left(t-T_{c} / 2\right)
\end{aligned}
$$

where $\gamma \in[0, \infty)$ is a parameter of the chip-weighting waveforms. When $\gamma=0$ in (7), the chip-weighting waveforms $c w_{p}(t)$ for all $p \in[1,2,3,4]$ reduce to the rectangular pulse $P_{T_{c}}(t)$. Fig. 2 shows the chip-weighting waveforms and Fig. 3 shows waveforms of length-14 segments of a spreading sequence and the corresponding weighted despreading sequences for three values of $\gamma$. As pointed out in [15], the reason why the weighted despreading sequence helps to reject the MAI is that the weighted sequence makes use of the property that the occurrence of the transitions in the reference signal is independent of that in MAI signals. The weighted despreading sequence emphasizes the transitions in the desired spreading signal. Because MAI signals arrive with random delay, their transitions are less likely to be emphasized, and this leads to the MAI rejection at the output of the integrate-and-dump circuit.

For DPSK modulation, the structure of path $l$ of user $k$ 's RAKE demodulator using noncoherent detection is shown in Fig. 4. In this case, the outputs of all single-path receiver $\operatorname{Re}\left[\vartheta_{k l}(\kappa) \vartheta_{k l}^{*}(\kappa-1)\right], l=0,1, \cdots, L_{R}-1$, are directly summed to form a single decision variable $\vartheta_{k}(\kappa)$. Note that the weighted despreading sequences are also used in the RAKE demodulator for MAI rejection.

\section{System PERformance}

For ease in analysis, it is assumed that the average fading power for each path of any user's channel is identical so that the average fading powers $\rho_{k l}=\rho(1 \leq k \leq K$, $\left.0 \leq l \leq L_{P}-1\right)$ are independent of $k$ and $l$.
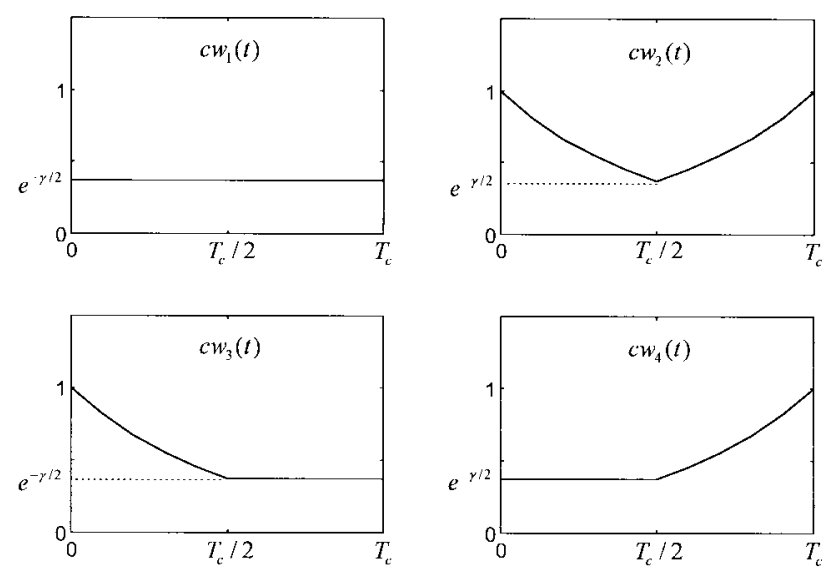

Fig. 2. The exponential chip-weighting waveforms for despreading sequences.

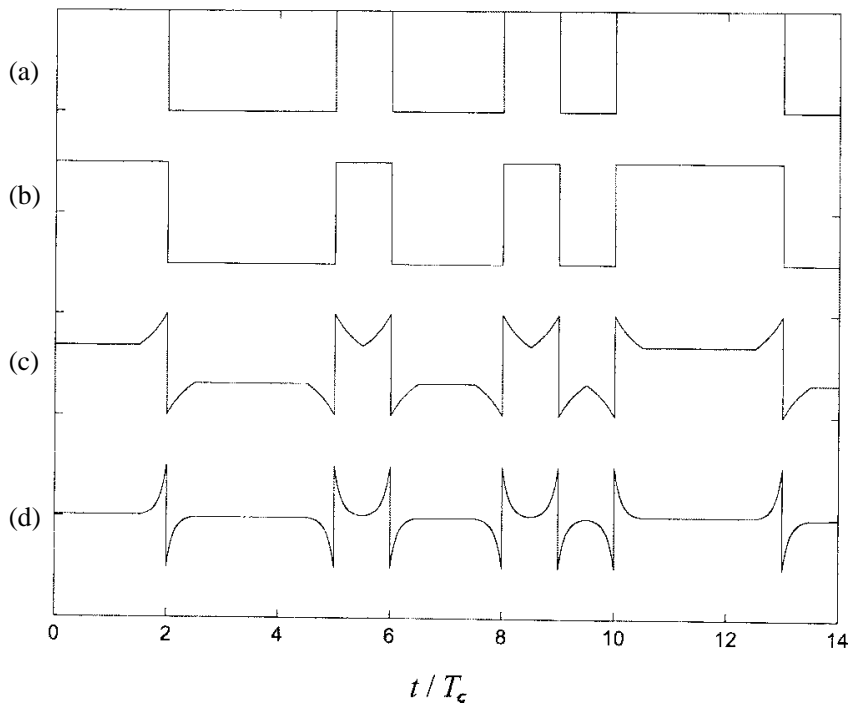

Fig. 3. Waveforms of length-14 segments of a spreading sequence and the corresponding weighted despreading sequences (WDS): (a) spreading sequence, (b) WDS with $\gamma=0$, (c) WDS with $\gamma=2$, and (d) WDS with $\gamma=8$.

\section{A. Coherent Reception}

We arbitrarily choose the $i$ th user as the reference user and analyze the performance of the RAKE receiver with coherent detection for data symbol $b_{\lambda}^{(i)}$. After the $l$ th path matched filter which has the impulse response matched to 


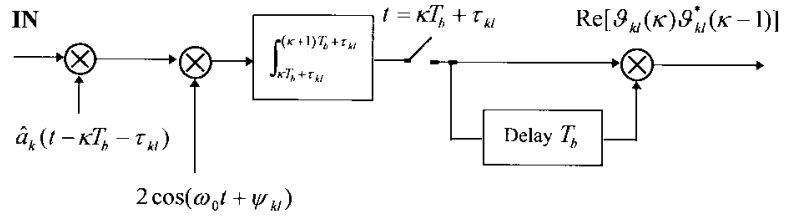

Fig. 4. The structure of path $l$ 's noncoherent receiver for the $k$ th user.

$2 \hat{a}_{i}(t) \cos \left(\omega_{0} t\right) P_{T_{b}}(t)$, the conditional output random variable, denoted by $\zeta_{i l}(\lambda)$, can be expressed as

$$
\begin{aligned}
\zeta_{i l}(\lambda)= & \int_{\lambda T_{b}+\tau_{i l}}^{(\lambda+1) T_{b}+\tau_{i l}} \\
& \cdot\left\{2 r(t) \hat{a}_{i}\left(t-\lambda T_{b}-\tau_{i l}\right) \cos \left(\omega_{0} t+\phi_{i l}\right)\right\} d t
\end{aligned}
$$

where $\cos \left(\omega_{0} t+\phi_{i l}\right)$ is the coherent carrier reference and $\hat{a}_{i}\left(t-\lambda T_{b}-\tau_{i l}\right)$ is the weighted despreading sequence for the $\lambda$ th bit of the $l$ th path signal of the reference user. Since the carrier frequency $f_{0}$ is much larger than $T_{b}^{-1}$ in practical systems, the double-frequency terms in (8) is ignored in our analysis. Then (8) is reduced to

$$
\zeta_{i l}(\lambda)=S_{i l}(\lambda)+N_{i l}(\lambda)+\sum_{\substack{k=1 \\ k \neq i}}^{K} \sum_{n=0}^{L_{P}-1} Y_{k, n, l}+\sum_{\substack{n=0 \\ n \neq l}}^{L_{P}-1} Y_{i, n, l}
$$

where the first, second, third, and forth components are the desired, AWGN, MAI, and self-generated multipath interference components which are described in detail below.

From (8), the desired signal term $S_{i l}(\lambda)$ for the $l$ th path matched filter can be written as

$$
\begin{aligned}
S_{i l}(\lambda)= & \sqrt{2 P} G_{i} \beta_{i l} b_{\lambda}^{(i)} \int_{\lambda T_{b}+\tau_{i l}}^{(\lambda+1) T_{b}+\tau_{i l}} \\
& \cdot a_{i}\left(t-\tau_{i l}\right) \hat{a}_{i}\left(t-\lambda T_{b}-\tau_{i l}\right) d t \\
= & \sqrt{2 P} G_{i} \beta_{i l} b_{\lambda}^{(i)} \sum_{j=0}^{N-1} \int_{j T_{c}}^{(j+1) T_{c}} \\
& \cdot w_{j}^{(i)}\left(t-j T_{c} \mid\left\{c_{j}^{(i)}, c_{j+1}^{(i)}\right\}\right) d t .
\end{aligned}
$$

Making a change of the integral limits in (10) and then using (6) and (7), the signal term $S_{i l}(\lambda)$ can be expressed as

$$
\begin{aligned}
S_{i l}(\lambda)= & b_{\lambda}^{(i)} \sqrt{2 P} \beta_{i l} G_{i}\left[2 \hat{N}_{i} \int_{0}^{T_{c} / 2} e^{-\gamma t / T_{c}} d t\right. \\
& \left.+\left(N-\hat{N}_{i}\right) \int_{0}^{T_{c}} e^{-\gamma / 2} d t\right] \\
= & b_{\lambda}^{(i)} \sqrt{2 P} \beta_{i l} G_{i}\left[2 \hat{N}_{i} T_{c}\left(1-e^{-\gamma / 2}\right) /\right. \\
& \left.\gamma+\left(N-\hat{N}_{i}\right) T_{c} e^{-\gamma / 2}\right]
\end{aligned}
$$

where $\hat{N}_{i}$ is a random variable which represents the number of times of occurrence that $c_{j}^{(i)}=-1$ for all $j \in[0, N-1]$. The desired signal term of the RAKE receiver $S_{i}(\lambda)$, which is the weighted sum of the outputs of the single-path matched filters, is then given by

$$
\begin{aligned}
S_{i}(\lambda)= & \sum_{l=0}^{L_{R}-1} \beta_{i l} S_{i l}(\lambda) \\
= & b_{\lambda}^{(i)} \sqrt{2 P} G_{i}\left[2 \hat{N}_{i} T_{c}\left(1-e^{-\gamma / 2}\right) /\right. \\
& \left.\gamma+\left(N-\hat{N}_{i}\right) T_{c} e^{-\gamma / 2}\right] \sum_{l=0}^{L_{R}-1} \beta_{i l}^{2} .
\end{aligned}
$$

$N_{i l}(\lambda)$ in (9) is the $l$ th path AWGN term and is given by

$$
N_{i l}(\lambda)=\int_{0}^{T_{b}}\left[n_{c}(t) \cos \phi_{i l}-n_{s}(t) \sin \phi_{i l}\right] \hat{a}_{i}(t) d t .
$$

Using the approach that leads to (11), the variance of the noise term $N_{i l}(\lambda)$, conditioned on $\left\{c_{j}^{(i)}\right\}$, is given by

$$
\begin{aligned}
\operatorname{var} & {\left[N_{i l}(\lambda)\right] } \\
& =N_{0}\left[2 \hat{N}_{i} \int_{0}^{T_{c} / 2} e^{-2 \gamma t / T_{c}} d t+\left(N-\hat{N}_{i}\right) \int_{0}^{T_{c}} e^{-\gamma} d t\right] \\
& =N_{0}\left[\hat{N}_{i} T_{c}\left(1-e^{-\gamma}\right) / \gamma+\left(N-\hat{N}_{i}\right) T_{c} e^{-\gamma}\right] .
\end{aligned}
$$

The total AWGN in the decision variable of the $i$ th RAKE receiver, denoted by $N_{i}(\lambda)$, can be expressed as

$$
N_{i}(\lambda)=\sum_{l=0}^{L_{R}-1} \beta_{i l} N_{i l}(\lambda)
$$

Because each of the single-path matched filters is matched to a unique phase and different phases are independent random variables distributed uniformly over $[0,2 \pi]$, the elements of the outputs of the single-path matched filters caused by MAI and AWGN are uncorrelated among each other. This can be proved by similar approach used in [11, Appendix B] so that we assume that the different path noise terms, including MAI and AWGN, are uncorrelated with each other in what follows. Thus, the variance of the total AWGN $N_{i}(\lambda)$ of the $i$ th RAKE receiver, conditioned on $\left\{\beta_{i l}\right\}$ and $\left\{c_{j}^{(i)}\right\}$ and denoted by $\sigma_{N \mid\left\{\beta_{i l}, c_{j}^{(i)}\right\}}^{2}$, is given by

$$
\begin{aligned}
& \sigma_{N \mid\left\{\beta_{i l}, c_{j}^{(i)}\right\}}^{L_{R}-1} \beta_{i l}^{2} \operatorname{var}\left[N_{i l}(\lambda)\right] \\
& \quad=\sum_{l=0}^{L_{L^{\prime}}}{ }^{2} N_{0}\left[\hat{N}_{i} T_{c}\left(1-e^{-\gamma}\right) / \gamma+\left(N-\hat{N}_{i}\right) T_{c} e^{-\gamma}\right] \sum_{l=0}^{L_{R}-1} \beta_{i l}^{2} .
\end{aligned}
$$

The third term in (9) is the MAI term of the $l$ th single-path matched filter of the $i$ th RAKE receiver and $Y_{k, n, l}$ can be expressed as

$$
\begin{aligned}
Y_{k, n, l}= & \sqrt{2 P} G_{k} \beta_{k n} \cos \left(\phi_{i l}-\phi_{k n}\right) \\
& \cdot\left[b_{\lambda-1}^{(k)} R_{i k}\left(\tau_{n l}^{(k, i)}\right)+b_{\lambda}^{(k)} \hat{R}_{i k}\left(\tau_{n l}^{(k, i)}\right)\right]
\end{aligned}
$$


where $\tau_{n l}^{(k, i)}=\tau_{k n}-\tau_{i l}, R_{i k}(\tau)=\int_{0}^{\tau} a_{k}(t-\tau) \hat{a}_{i}(t) d t$, and $\hat{R}_{i k}(\tau)=\int_{\tau}^{T_{b}} a_{k}(t-\tau) \hat{a}_{i}(t) d t$. The variance of the term $Y_{k, n, l}$, conditioned on $\left\{c_{j}^{(i)}\right\}$, can be expressed in the form

$$
\begin{aligned}
\operatorname{var}\left[Y_{k, n, l}\right] & =E\left(Y_{k, n, l}^{2} \mid\left\{c_{j}^{(i)}\right\}\right) \\
& =2 \rho P\left(1+\frac{\varepsilon_{m}^{2}}{3}\right)\left\{\bar{\Theta}_{\left\{c_{j}^{(i)}\right\}}\left(R_{i k}, \hat{R}_{i k}\right)-\mu_{i k \mid\left\{c_{j}^{(i)}\right\}}\right\}
\end{aligned}
$$

where

$$
\begin{aligned}
& \bar{\Theta}_{\left\{c_{j}^{(i)}\right\}}\left(R_{i k}, \hat{R}_{i k}\right) \\
& \quad=E\left\{\left.\left[R_{i k}\left(\tau_{n l}^{(k, i)}\right)+\hat{R}_{i k}\left(\tau_{n l}^{(k, i)}\right)\right]^{2}\right|_{\left\{c_{j}^{(i)}\right\}}\right\}
\end{aligned}
$$

and

$$
\mu_{i k \mid\left\{c_{j}^{(i)}\right\}}=2 E\left[\left.R_{i k}\left(\tau_{n l}^{(k, i)}\right) \hat{R}_{i k}\left(\tau_{n l}^{(k, i)}\right)\right|_{\left\{c_{j}^{(i)}\right\}}\right]
$$

Both $\bar{\Theta}_{\left\{c_{j}^{(i)}\right\}}\left(R_{i k}, \hat{R}_{i k}\right)$ and $\mu_{i k \mid\left\{c_{j}^{(i)}\right\}}$ are conditioned on $\left\{c_{j}^{(i)}\right\}$. According to the results derived in [18], (18) can be approximately written as

$$
\begin{aligned}
& \operatorname{var}\left[Y_{k, n, l}\right] \\
& \quad \approx 2 \rho P\left(1+\frac{\varepsilon_{m}^{2}}{3}\right)\left(\frac{N T_{c}^{2}}{\gamma^{2}} e^{-\gamma}\right) \Xi^{(e)}\left(\Gamma^{\left\{c_{j}^{(i)}\right\}}, \gamma\right)
\end{aligned}
$$

where

$$
\begin{aligned}
\Xi^{(e)} & \left(\Gamma^{\left\{c_{j}^{(i)}\right\}}, \gamma\right) \\
= & \frac{1}{N}\left\{\Gamma_{\{-1,-1,-1\}}^{(i)}\left[4+\frac{12}{\gamma}-\frac{16}{\gamma} e^{\gamma / 2}+\frac{4}{\gamma} e^{\gamma}\right]\right. \\
& +\left(\Gamma_{\{-1,-1,1\}}^{(i)}+\Gamma_{\{1,-1,-1\}}^{(i)}\right) \\
& \cdot\left[\frac{5}{2}-\frac{\gamma}{4}+\frac{\gamma^{2}}{24}+\frac{19}{2 \gamma}+e^{\gamma / 2}-\frac{12}{\gamma} e^{\gamma / 2}+\frac{5}{2 \gamma} e^{\gamma}\right] \\
& +\left(\Gamma_{\{-1,1,1\}}^{(i)}+\Gamma_{\{1,1,-1\}}^{(i)}\right) \\
& \cdot\left[-\frac{3}{2}-\frac{3}{4} \gamma+\frac{19}{24} \gamma^{2}-\frac{1}{2 \gamma}+e^{\gamma / 2}+\frac{e^{\gamma}}{2 \gamma}\right] \\
& +\Gamma_{\{-1,1,-1\}}^{(i)}\left[-3-\frac{3}{2} \gamma+\frac{7}{12} \gamma^{2}-\frac{1}{\gamma}+2 e^{\gamma / 2}+\frac{e^{\gamma}}{\gamma}\right] \\
& +\Gamma_{\{1,-1,1\}}^{(i)}\left[1-\frac{\gamma}{2}+\frac{\gamma^{2}}{12}+\frac{7}{\gamma}+2 e^{\gamma / 2}-\frac{8}{\gamma} e^{\gamma / 2}+\frac{e^{\gamma}}{\gamma}\right] \\
& \left.+\gamma^{2} \Gamma_{\{1,1,1\}}^{(i)}\right\} .
\end{aligned}
$$

$\Gamma_{\left\{v_{1}, v_{2}, v_{3}\right\}}^{(i)}$ is the number of times of occurrence that $\left\{c_{j-1}^{(i)}\right.$, $\left.c_{j}^{(i)}, c_{j+1}^{(i)}\right\}=\left\{v_{1}, v_{2}, v_{3}\right\}$ for all $j \in[0, N-1]$ in the $i$ th weighted despreading sequence and each element of $\left\{v_{1}, v_{2}\right.$, $v_{3}$ \} takes values +1 or -1 with equal probabilities. It is clear that $\sum_{\left\{v_{1}, v_{2}, v_{3}\right\}} \Gamma_{\left\{v_{1}, v_{2}, v_{3}\right\}}^{(i)}=N$.

The total MAI of the $i$ th RAKE receiver $I_{i, k}$, which is the weighted sum of the MAI terms of the single-path matched filters, may be expressed as

$$
I_{i, k}=\sum_{l=0}^{L_{R}-1} \beta_{i l} \sum_{\substack{k=1 \\ k \neq i}}^{K} \sum_{n=0}^{L_{P}-1} Y_{k, n, l} .
$$

As mentioned previously, the elements of the outputs of the single-path matched filters caused by MAI are uncorrelated so that the variance of the total MAI $I_{i, k}$, denoted by $\sigma_{M \mid\left\{\beta_{i l}, c_{j}^{(i)}\right\}}^{2}$ and conditioned on $\left\{\beta_{i l}\right\}$ and $\left\{c_{j}^{(i)}\right\}$, is then given by

$$
\begin{aligned}
\sigma_{M \mid\left\{\beta_{i l}, c_{j}^{(i)}\right\}}^{2}= & E\left(I_{i, k}^{2} \mid\left\{\beta_{i l}\right\},\left\{c_{j}^{(i)}\right\}\right) \\
= & L_{p}(K-1) \operatorname{var}\left[Y_{k, n, l}\right] \sum_{l=0}^{L_{R}-1} \beta_{i l}^{2} \\
= & 2 \rho P L_{p}(K-1)\left(1+\frac{\varepsilon_{m}^{2}}{3}\right)\left(\frac{N T_{c}^{2}}{\gamma^{2}} e^{-\gamma}\right) \\
& \cdot \Xi^{(e)}\left(\Gamma^{\left\{c_{j}^{(i)}\right\}}, \gamma\right) \sum_{l=0}^{L_{R}-1} \beta_{i l}^{2} .
\end{aligned}
$$

The fourth term in (8) is the self-generated multipath interference term of the $l$ th single-path matched filter and the total self-interference of the $i$ th RAKE receiver, denoted by $I_{i, i}$, is given by

$$
\begin{aligned}
I_{i, i}= & \sum_{l=0}^{L_{R}-1} \beta_{i l} \sum_{n=0, n \neq l}^{L_{P}-1} Y_{i, n, l} \\
= & \sum_{l=0}^{L_{R}-1} \beta_{i l} \sum_{n=0, n \neq l}^{L_{P}-1} \sqrt{2 P} G_{i} \beta_{i n} \cos \left(\phi_{i l}-\phi_{i n}\right) \\
& \cdot\left[b_{\lambda-1}^{(i)} R_{i i}\left(\tau_{n l}^{(i, i)}\right)+b_{\lambda}^{(i)} \hat{R}_{i i}\left(\tau_{n l}^{(i, i)}\right)\right] .
\end{aligned}
$$

The term $I_{i, i}$ does not have a significant effect on performance comparison because of the fact that the self-generated interference will have much less power than the MAI when the number of active users $K \gg 1$. However, its precise effect on the system performance is much more difficult to analyze than the other terms in (9). For simplicity, some modification to this term must be made. One way is to simply ignore it for selected spreading sequences [12], [19], [20] and another is to treat it crudely as one additional user for more pessimistic results [10], [11]. We evaluate the conditional variance of this term by taking ensemble average over all random variables of the reference user except $\left\{\beta_{i l}\right\}$. Thus, the variance of the self-generated multipath interference $\sigma_{S \mid\left\{\beta_{i l}\right\}}^{2}$, conditioned on $\left\{\beta_{i l}\right\}$, may be expressed as

$$
\begin{aligned}
\sigma_{S \mid\left\{\beta_{i l}\right\}}^{2}= & E\left(I_{i, i}^{2} \mid\left\{\beta_{i l}\right\}\right) \\
= & 2 \rho P\left(\frac{1+\varepsilon_{m}^{2}}{3}\right)\left(\sum_{n=0, n \neq l}^{L_{P}-1}\left\{\bar{\Theta}\left(R_{i i}, \hat{R}_{i i}\right)-\mu_{i i}\right\}\right) \\
& \cdot \sum_{l=0}^{L_{R}-1} \beta_{i l}^{2}
\end{aligned}
$$


where

and

$$
\bar{\Theta}\left(R_{i i}, \hat{R}_{i i}\right)=E\left\{\left[R_{i i}\left(\tau_{n l}^{(i, i)}\right)+\hat{R}_{i i}\left(\tau_{n l}^{(i, i)}\right)\right]^{2}\right\}
$$

$$
\mu_{i i}=2 E\left[R_{i i}\left(\tau_{n l}^{(i, i)}\right) \hat{R}_{i i}\left(\tau_{n l}^{(i, i)}\right)\right]
$$

Let $\tau_{n l}^{(i, i)}=m T_{c}-\tau_{0}$, where $\tau_{0}$ is a random variable distributed over $\left(0, T_{c}\right]$, and the integer $m \in[3, N-1]$ due to the assumption that the delay differences between the different paths of the signals of the reference user, $\tau_{n l}^{(i, i)}(n \neq l)$, are larger than $2 T_{c}$, we have

$$
\begin{aligned}
& R_{i i}\left(\tau_{n l}^{(i, i)}\right)+\hat{R}_{i i}\left(\tau_{n l}^{(i, i)}\right) \\
& \quad=\sum_{j=0}^{N-1} a_{N-m+j}^{(i)} a_{j}^{(i)} f\left(\tau_{0} \mid\left\{c_{j-1}^{(i)}, c_{j}^{(i)}, c_{j+1}^{(i)}\right\}\right)
\end{aligned}
$$

where

$$
\begin{aligned}
f\left(\tau_{0} \mid\right. & \left.\left\{c_{j-1}^{(i)}, c_{j}^{(i)}, c_{j+1}^{(i)}\right\}\right) \\
= & c_{j}^{(i)} \int_{T_{c}-\tau_{0}}^{T_{c}} w_{j-1}^{(i)}\left(t \mid\left\{c_{j-1}^{(i)}, c_{j}^{(i)}\right\}\right) d t \\
& +\int_{0}^{T_{c}-\tau_{0}} w_{j}^{(i)}\left(t \mid\left\{c_{j}^{(i)}, c_{j+1}^{(i)}\right\}\right) d t .
\end{aligned}
$$

It is clear that $E\left[R_{i i}\left(\tau_{n l}^{(i, i)}\right)+\hat{R}_{i i}\left(\tau_{n l}^{(i, i)}\right)\right]=0$ when $m \in$ $[3, N-1]$. Thus, the variance of the self-generated multipath interference is approximately given by [18]

$$
\begin{aligned}
\sigma_{S \mid\left\{\beta_{i l}\right\}}^{2} \approx & 2 \rho P\left(L_{P}-1\right)\left(1+\frac{\varepsilon_{m}^{2}}{3}\right)\left(\frac{N T_{c}^{2}}{\gamma^{2}} e^{-\gamma} \bar{\Xi}^{(e)}(\gamma)\right) \\
& \cdot \sum_{l=0}^{L_{R}-1} \beta_{i l}^{2}
\end{aligned}
$$

where

$$
\begin{aligned}
& \bar{\Xi}^{(e)}(\gamma) \\
& =\left(\frac{1}{2}-\frac{\gamma}{2}+\frac{5 \gamma^{2}}{12}+\frac{9}{2 \gamma}+e^{\gamma / 2}-\frac{6}{\gamma} e^{\gamma / 2}+\frac{3}{2 \gamma} e^{\gamma}\right) .
\end{aligned}
$$

Note that $E_{c_{j}^{(i)}}\left[\Xi^{(e)}\left(\Gamma^{\left\{c_{j}^{(i)}\right\}}, \gamma\right)\right]=\bar{\Xi}^{(e)}(\gamma)$ where $E_{c_{j}^{(i)}}[\cdot]$ denotes ensemble average over all $c_{j}^{(i)}$ for $-\infty<j<+\infty$. Since there should be relatively little difference between $\Xi^{(e)}\left(\Gamma^{\left\{c_{j}^{(i)}\right\}}, \gamma\right)$ and $\Xi^{(e)}(\gamma)$ when the processing gain $N$ is large, coupled with the fact that $\sigma_{M \mid\left\{\beta_{i l}, c_{j}^{(i)}\right\}}^{2} \gg \sigma_{S \mid\left\{\beta_{i l}\right\}}^{2}$ when the number of active users $K \gg 1$, the term $\bar{\Xi}^{(e)}(\gamma)$ in (27) can be replaced by $\Xi^{(e)}\left(\Gamma^{\left\{c_{j}^{(i)}\right\}}, \gamma\right)$ with a negligible effect on the total variance of interference.

Thus, the variance of the total interference $\sigma_{T \mid\left\{\beta_{i l}, c_{j}^{(i)}\right\}}^{2}$ is approximately given by

$$
\begin{aligned}
\sigma_{T \mid\left\{\beta_{i l}, c_{j}^{(i)}\right\}}^{2}= & \sigma_{M \mid\left\{\beta_{i l}, c_{j}^{(i)}\right\}}^{2}+\sigma_{S \mid\left\{\beta_{i l}\right\}}^{2} \\
\approx & 2 \rho P\left(K L_{P}-1\right)\left(1+\frac{\varepsilon_{m}^{2}}{3}\right)\left(\frac{N T_{c}^{2}}{\gamma^{2}} e^{-\gamma}\right) \\
& \cdot \Xi^{(e)}\left(\Gamma^{\left\{c_{j}^{(i)}\right\}}, \gamma\right) \sum_{l=0}^{L_{R}-1} \beta_{i l}^{2}
\end{aligned}
$$

By definition, the total conditional signal to interference plus noise ratio $\mathrm{SINR}_{i}$ of the decision variable of the reference RAKE receiver may be expressed in the form

$$
\mathrm{SINR}_{i}=\left(\frac{\sigma_{N \mid\left\{\beta_{i l}, c_{j}^{(i)}\right\}}^{2}}{S_{i}^{2}(\lambda)}+\frac{\sigma_{T \mid\left\{\beta_{i l}, c_{j}^{(i)}\right\}}^{2}}{S_{i}^{2}(\lambda)}\right)^{-1}
$$

where $S_{i}(\lambda), \sigma_{N \mid\left\{\beta_{i l}, c_{j}^{(i)}\right\}}^{2}$ and $\sigma_{T \mid\left\{\beta_{i l}, c_{j}^{(i)}\right\}}^{2}$ are given by (12), (16), and (29), respectively.

Because the MAI is the sum of many independent couser signals, it can be modeled as conditional zero-mean Gaussian process. Gaussian approximation method has been used in the study of similar models (e.g., [5], [6], and [9]) and has been reported to be accurate even for moderate number of cousers. Using this approach the conditional error probability $P_{e \mid\left\{\beta_{i l}\right\}, G_{i}}^{(i)}$, conditioned on $\left\{\beta_{i l}\right\}, G_{i}$, and $\left\{c_{j}^{(i)}\right\}$, is given by

$$
P_{e \mid\left\{\beta_{i l}\right\}, G_{i}}^{(i)}=Q\left(\sqrt{\mathrm{SINR}_{i}}\right)
$$

where

$$
Q(x)=\frac{1}{\sqrt{2 \pi}} \int_{x}^{\infty} e^{-t^{2} / 2} d t
$$

It is well known that the RAKE receiver under consideration is equivalent to a receiver with maximal ratio combiner [21]. Since it is assumed that $E\left[\beta_{i l}^{2}\right]=2 \rho$ for all paths and users, the conditional error probability $P_{e \mid G_{i}}^{(i)}$ conditioned on $G_{i}$ and $\left\{c_{j}^{(i)}\right\}$ is then given by [21, eq. (7.4.15)]

$$
P_{e \mid G_{i}}^{(i)}=\left(\frac{1-\mu}{2}\right)^{L_{R}} \sum_{k=0}^{L_{R}-1}\left(\begin{array}{c}
L_{R}-1+k \\
k
\end{array}\right)\left(\frac{1+\mu}{2}\right)^{k}
$$

where

$$
\begin{aligned}
\mu & =\sqrt{\frac{R_{c}}{1+R_{c}}} \\
R_{c} & =\hat{H}^{2} G_{i}^{2}
\end{aligned}
$$

and $\hat{H}$ is the average signal to interference plus noise ratio per channel, given by

$$
\begin{aligned}
\hat{H}=\left\{\frac{\gamma\left[\chi\left(1-e^{-\gamma}\right)+\gamma(1-\chi) e^{-\gamma}\right]}{\bar{\gamma}_{b}\left[2 \chi\left(1-e^{-\gamma / 2}\right)+\gamma(1-\chi) e^{-\gamma / 2}\right]^{2}}\right. \\
\\
\left.+\frac{\left(K L_{P}-1\right)\left(1+\varepsilon_{m}^{2} / 3\right) \Xi^{(e)}\left(\Gamma^{\left\{c_{j}^{(i)}\right\}}, \gamma\right)}{N\left[2 \chi\left(e^{\gamma / 2}-1\right)+\gamma(1-\chi)\right]^{2}}\right\}
\end{aligned}
$$

with $\chi=\hat{N}_{i} / N, \bar{\gamma}_{b}=\bar{E}_{b} / N_{0}$, and $\bar{E}_{b}=2 \rho P T_{b}$. In order to remove the conditional probability on $G_{i}$ in (33), and obtain $P_{e}^{(i)}$, we evaluate the integral

$$
P_{e}^{(i)}=\frac{1}{2 \varepsilon_{m}} \int_{1-\varepsilon_{m}}^{1+\varepsilon_{m}} P_{e \mid G_{i}}^{(i)} d G_{i}
$$


According to the Appendix, a closed-form solution for (37) is given by

$$
\begin{aligned}
P_{e}^{(i)}= & \frac{1}{2}-\sum_{d=1}^{L_{R}} \frac{\left(\begin{array}{c}
2 d-2 \\
d-1
\end{array}\right)}{2^{2 d} \varepsilon_{m} \hat{H}(2 d-3)} \\
& \cdot\left\{\left(b^{2}+1\right)^{(3-2 d) / 2}-\left(a^{2}+1\right)^{(3-2 d) / 2}\right\}
\end{aligned}
$$

where $a=\left(1+\varepsilon_{m}\right) \hat{H}$ and $b=\left(1-\varepsilon_{m}\right) \hat{H}$. Equation (38) will enable us to compute the BER for coherent reception at a given set of system parameters.

\section{B. Noncoherent Reception}

For noncoherent detection, the RAKE structure receiver for DPSK signals is called RAKE demodulator. After the $l$ th path receiver of the $i$ th RAKE demodulator, the random variable $\hat{\vartheta}_{i l}(\lambda)$ for data symbol $b_{\lambda}^{(i)}$ can be written as

$$
\hat{\vartheta}_{i l}(\lambda)=\operatorname{Re}\left[\vartheta_{i l}(\lambda) \vartheta_{i l}^{*}(\lambda-1)\right]
$$

where $\operatorname{Re}[\cdot]$ stands for the "real part," * denotes complex conjugate, and

$$
\begin{array}{r}
\vartheta_{i l}(\lambda)=\int_{\lambda T_{b}+\tau_{i l}}^{(\lambda+1) T_{b}+\tau_{i l}}\left\{2 r(t) \hat{a}\left(t-\lambda T_{b}-\tau_{i l}\right)\right. \\
\left.\cdot \exp \left(j \omega_{0} t+j \psi_{i l}\right)\right\} d t
\end{array}
$$

where $\psi_{i l}$ is a random phase. Using the same argument in the derivation of (9) to drop the double-frequency components of $r(t) \exp \left(j \omega_{0} t+j \psi_{i l}\right)$, (40) becomes

$$
\vartheta_{i l}(\lambda)=D_{i l}(\lambda)+W_{i l}(\lambda)+\sum_{\substack{k=1 \\ k \neq i}}^{K} \sum_{n=0}^{L_{P}-1} J_{k, n, l}+\sum_{\substack{n=0 \\ n \neq l}}^{L_{P}-1} J_{i, n, l}
$$

where the terms on the right-hand side (RHS) of (41) are described in detail below.

For ease in computation, it is assumed that, without loss in generality, $\phi_{i l}=\psi_{i l}$ in (40). Therefore, similar to the derivation of (12), the desired signal term $D_{i l}(\lambda)$ in (41) can be expressed in the form

$$
\begin{aligned}
D_{i l}(\lambda)=b_{\lambda}^{(i)} \sqrt{2 P} G_{i} \beta_{i l}[ & {\left[2 \hat{N}_{i} T_{c}\left(1-e^{-\gamma / 2}\right) /\right.} \\
& \left.+\gamma\left(N-\hat{N}_{i}\right) T_{c} e^{-\gamma / 2}\right] .
\end{aligned}
$$

The second term in (41) is a complex-valued AWGN term. Following the approach used in the derivation of (16), the variance of the complex-valued AWGN term $\hat{\sigma}_{N \mid\left\{c_{j}^{(i)}\right\}}^{2}$, conditioned on $\left\{c_{j}^{(i)}\right\}$, is given by

$$
\hat{\sigma}_{N \mid\left\{c_{j}^{(i)}\right\}}^{2}=2 N_{0}\left[\hat{N}_{i} T_{c}\left(1-e^{-\gamma}\right) / \gamma+\left(N-\hat{N}_{i}\right) T_{c} e^{-\gamma}\right] .
$$

The third term in (41) is a complex-valued MAI term due to couser's signals. From (40), the complex-valued MAI, denoted by $\hat{I}_{i, k}$, can be written as

$$
\begin{aligned}
\hat{I}_{i, k}= & \sum_{\substack{k=1 \\
k \neq i}}^{K} \sum_{n=0}^{L_{P}-1} J_{k, n, l} \\
= & \sum_{\substack{k=1 \\
k \neq i}}^{K} \sum_{n=0}^{L_{P}-1} \sqrt{2 P} G_{k} \beta_{k n} \exp \left[j v_{n, l}^{(k, i)}\right] \\
& \cdot\left[b_{\lambda-1}^{(k)} R_{i k}\left(\tau_{n l}^{(k, i)}\right)+b_{\lambda}^{(k)} \hat{R}_{i k}\left(\tau_{n l}^{(k, i)}\right)\right]
\end{aligned}
$$

where $v_{n, l}^{(k, i)}=\phi_{k n}-\psi_{i l}$. Similar to the derivation of (19), the variance of the complex-valued MAI term $\hat{\sigma}_{M \mid\left\{c_{j}^{(i)}\right\}}^{2}$, conditioned on $\left\{c_{j}^{(i)}\right\}$, is given by

$$
\begin{aligned}
\hat{\sigma}_{M \mid\left\{c_{j}^{(i)}\right\}}^{2}= & E\left[\left|\hat{I}_{i, k}\right|^{2} \mid\left\{c_{j}^{(i)}\right\}\right] \\
= & 4 \rho P L_{P}(K-1)\left(1+\frac{\varepsilon_{m}^{2}}{3}\right)\left(\frac{N T_{c}^{2}}{\gamma^{2}} e^{-\gamma}\right) \\
& \cdot \Xi^{(e)}\left(\Gamma^{\left\{c_{j}^{(i)}\right\}}, \gamma\right) .
\end{aligned}
$$

The last term in (41) is the complex-valued self-generated interference term due to multipath signals of the reference user and can be expressed in the form

$$
\begin{aligned}
\hat{I}_{i, i}= & \sum_{\substack{n=0 \\
n \neq l}}^{L_{P}-1} J_{i, n, l} \\
= & \sum_{\substack{n=0 \\
n \neq l}}^{L_{P}-1} \sqrt{2 P} G_{i} \beta_{i n} \exp \left[j v_{n, l}^{(i, i)}\right] \\
& \cdot\left[b_{\lambda-1}^{(i)} R_{i i}\left(\tau_{n l}^{(i, i)}\right)+b_{\lambda}^{(i)} \hat{R}_{i i}\left(\tau_{n l}^{(i, i)}\right)\right] .
\end{aligned}
$$

By the same approximation used in the derivation of (27), the variance of the term $\hat{I}_{i, i}$, denoted by $\hat{\sigma}_{S}^{2}$, is given by

$$
\begin{aligned}
\hat{\sigma}_{S}^{2} & =E\left[\left|\hat{I}_{i, i}\right|^{2}\right] \\
& \approx 4 \rho P\left(L_{P}-1\right)\left(1+\frac{\varepsilon_{m}^{2}}{3}\right)\left(\frac{N T_{c}^{2}}{\gamma^{2}} e^{-\gamma} \bar{\Xi}^{(e)}(\gamma)\right) .
\end{aligned}
$$

Based on the fact that $\hat{\sigma}_{M \mid\left\{c_{j}^{(i)}\right\}}^{2} \gg \hat{\sigma}_{S}^{2}$ when $K \gg 1$ and $\Xi^{(e)}\left(\Gamma^{\left\{c_{j}^{(i)}\right\}}, \gamma\right) \approx \bar{\Xi}^{(e)}(\gamma)$ for large $N$, the total interference in (41) has a conditional variance $\hat{\sigma}_{T \mid\left\{c_{j}^{(i)}\right\}}^{2}$ approximately equal to

$$
\begin{aligned}
\hat{\sigma}_{T \mid\left\{c_{j}^{(i)}\right\}}^{2}= & \hat{\sigma}_{M \mid\left\{c_{j}^{(i)}\right\}}^{2}+\hat{\sigma}_{S}^{2} \\
\approx & 4 \rho P\left(K L_{P}-1\right)\left(1+\frac{\varepsilon_{m}^{2}}{3}\right) \\
& \cdot\left(\frac{N T_{c}^{2}}{\gamma^{2}} e^{-\gamma} \bar{\Xi}^{(e)}\left(\Gamma^{\left\{c_{j}^{(i)}\right\}}, \gamma\right)\right) .
\end{aligned}
$$


The decision variable of the reference RAKE demodulator $\vartheta_{i}(\lambda)$ is the direct sum of $\operatorname{Re}\left[\vartheta_{i l}(\lambda) \vartheta_{i l}^{*}(\lambda-1)\right]$, given by

$$
\vartheta_{i}(\lambda)=\sum_{l=0}^{L_{R}-1} \operatorname{Re}\left[\vartheta_{i l}(\lambda) \vartheta_{i l}^{*}(\lambda-1)\right], \quad\left(1 \leq L_{R} \leq L_{P}\right) .
$$

The conditional probability of error $\hat{P}_{e \mid\left\{\beta_{i l}\right\}, G_{i}}^{(i)}$, conditioned on $\left\{\beta_{i l}\right\}, G_{i}$, and $\left\{c_{j}^{(i)}\right\}$, is simply the probability that either $\vartheta_{i}(\lambda)<0$ or $\vartheta_{i}(\lambda)>0$. According to [21, eq. (4.4.13)], we have the conditional probability

$$
\hat{P}_{e \mid\left\{\beta_{i l}\right\}, G_{i}}^{(i)}=\frac{1}{2^{2 L_{R}-1}} e^{-\hat{r}_{d}} \sum_{n=0}^{L_{R}-1} c_{n} \hat{r}_{d}^{k}
$$

where

$$
c_{n}=\frac{1}{n !} \sum_{k=0}^{L_{R}-1-n}\left(\begin{array}{c}
2 L_{R}-1 \\
k
\end{array}\right)
$$

and $\hat{r}_{d}$ is the total conditional signal to interference plus noise ratio per bit for DPSK, given by

$$
\hat{r}_{d}=\frac{\sum_{l=0}^{L_{R}-1} D_{i l}^{2}(\lambda)}{\hat{\sigma}_{N \mid\left\{c_{j}^{(i)}\right\}}^{2}+\hat{\sigma}_{T \mid\left\{c_{j}^{(i)}\right\}}^{2}}
$$

where $D_{i l}(\lambda), \hat{\sigma}_{N \mid\left\{c_{j}^{(i)}\right\}}^{2}$, and $\hat{\sigma}_{T \mid\left\{c_{j}^{(i)}\right\}}^{2}$ are given by (42), (43), and (48), respectively. As indicated in [21], the conditional error probability $\hat{P}_{e \mid G_{i}}^{(i)}$ for binary DPSK signaling, conditioned on $\left\{c_{j}^{(i)}\right\}$ and $G_{i}$, can be manipulated into the same form given in (33) except that the variable $\mu$ in (34) is given by

$$
\mu=\frac{R_{c}}{1+R_{c}} .
$$

To obtain $\hat{P}_{e}^{(i)}$, which is only conditioned on $\left\{c_{j}^{(i)}\right\}$, the conditional error probability $\hat{P}_{e \mid G_{i}}^{(i)}$ must be averaged over the power control error statistics. Thus, we evaluate the integral

$$
\begin{aligned}
\hat{P}_{e}^{(i)}= & \left(\frac{1}{2 \varepsilon_{m}}\right) \sum_{k=0}^{L_{R}-1}\left(\begin{array}{c}
L_{R}-1+k \\
k
\end{array}\right) \int_{1-\varepsilon_{m}}^{1+\varepsilon_{m}} \\
& \cdot\left[\frac{1}{2\left(R_{c}+1\right)}\right]^{L_{R}}\left[\frac{1+2 R_{c}}{2\left(R_{c}+1\right)}\right]^{k} d G_{i}
\end{aligned}
$$

and the result is easily shown in (55), given at the bottom of the page. BER performance for noncoherent reception can be computed using (55) given a set of system parameters.

Based on [21, eq. (7.4.30)], we would like to point out that in a DS-CDMA system with power control error, the error probability given by (55) also applies to RAKE receiver with square-law combining for selected orthogonal spreading sequences by simply writing $\hat{H} / \sqrt{2}$ in place of $\hat{H}$. The configuration of the RAKE receiver can be found in [7, Fig. 2].

\section{Effects of System Condition on the Results}

Finally, a discussion on the effects of different system conditions on the results derived in this section is given. For noncoherent reception, it can be seen from (42), (43), and (48) that the signal term $D_{i l}(\lambda)$, the variance of white noise $\hat{\sigma}_{N \mid\left\{c_{j}^{(i)}\right\}}^{2}$ and the variance of the total MAI $\hat{\sigma}_{T \mid\left\{c_{j}^{(i)}\right\}}^{2}$ for the data symbol $b_{\lambda}^{(i)}$ are conditioned on either $\hat{N}_{i}$ or the elements of the set $\left\{\Gamma_{\{v 1, v 2, v 3\}}^{(i)}\right\}$. To simplify the analysis, we make the assumption that the spreading sequence of each user has a period equal to the processing gain $N$. It will ensure that either $\hat{N}_{i}$ or the elements of the set $\left\{\Gamma_{\{v 1, v 2, v 3\}}^{(i)}\right\}$ in $D_{i l}(\lambda)$, $\hat{\sigma}_{N \mid\left\{c_{j}^{(i)}\right\}}^{2}$, and $\hat{\sigma}_{T \mid\left\{c_{j}^{(i)}\right\}}^{2}$ for the data symbol $b_{\lambda}^{(i)}$ are identical to those for the previous data symbol $b_{\lambda-1}^{(i)}$ [22]. However, when the multipath delay spread of a fading channel is larger than the symbol duration, both self-interference and MAI will be increased significantly in the system under consideration. In order to reduce the interference caused by the multipath signals with delays longer than the symbol duration, the spreading sequences with period equal to $M N$ must be employed where the integer $M \gg 1$. Then the impulse responses of the singlepath matched filters of the reference user for both coherent and noncoherent receptions, which match to the same $T_{b}$-second segment of $\sum_{m=1}^{M} 2 \hat{a}_{i}(t) \cos \left(\omega_{0} t\right) P_{T_{b}}\left(t-m T_{b}\right)$, need to be updated every $T_{b}$ s [11], [12], [23]. If the $M$ subsequences are selected from all possible random sequences of length $N$ under the condition that all of the subsequences have the same $\hat{N}_{i}$ and the elements of the set $\left\{\Gamma_{\{v 1, v 2, v 3\}}^{(i)}\right\}$, the performance analysis taken in this section is not dependent on whether or not the single-path matched filters update their impulse responses every $T_{b} \mathrm{~s}$. The derived results therefore remain unchanged for the spreading sequences with period much longer than the processing gain $N$. For ease in analysis, we have assumed that all the fading paths have the same

$$
\hat{P}_{e}^{(i)}= \begin{cases}\left(\frac{1}{4 \varepsilon_{m} \hat{H}}\right) \operatorname{arctg}\left(\frac{a-b}{1+a b}\right), & L_{R}=1 \\
\left(\frac{1}{2 \varepsilon_{m} \hat{H}}\right) \sum_{k=0}^{L_{R}-1}\left(\begin{array}{c}
L_{R}-1+k \\
k
\end{array}\right) \sum_{q=L_{R}}^{L_{R}+k}(-1)^{q-L_{R}}\left(\frac{1}{2}\right)^{q}\left(\begin{array}{c}
k \\
q-L_{R}
\end{array}\right) & \\
\cdot\left\{\left(\frac{1}{2^{2 q-3}}\right)\left(\begin{array}{c}
2 q-3 \\
q-1
\end{array}\right) \operatorname{arctg}\left(\frac{a-b}{1+a b}\right)+\frac{1}{2 q-1} \sum_{n=1}^{q-1}\left[\frac{q}{(q-n) 2^{2 n}}\right]\right. & \\
\left.\cdot\left(\begin{array}{c}
2 q \\
q
\end{array}\right)\left[\frac{a}{\left(a^{2}+1\right)^{q-n}}-\frac{b}{\left(b^{2}+1\right)^{q-n}}\right] /\left(\begin{array}{c}
2 q-2 n \\
q-n
\end{array}\right)\right\}, & L_{R} \geq 2\end{cases}
$$




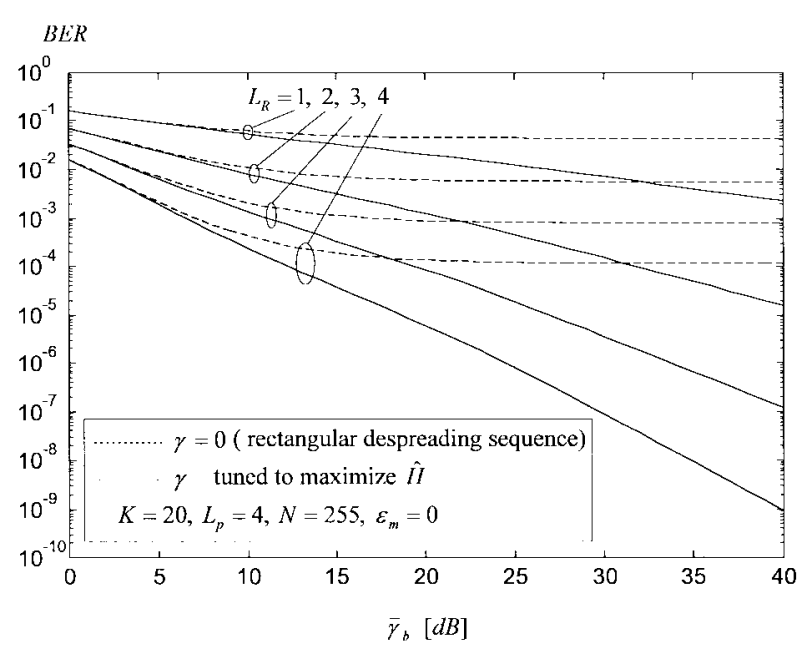

Fig. 5. BER's versus the average signal-to-noise ratio $\bar{\gamma}_{b}$ for RAKE receiver with different order of diversity.

average power. However, several measurements indicate that the multipath intensity profile (MIP) for a actual channel degrades as the path delay increases [24], [25]. Therefore, the diversity gain obtained from our equations becomes optimistic as the diversity order increases. The effects of the decayed MIP on diversity gain are shown clearly in [10] and [23].

\section{NumericAl Results AND Discussion}

In this section, we present numerical results on the BER performance of a DS-CDMA system with power control error using RAKE receptions over a multipath Rayleigh fading channel. The RAKE receivers under consideration employ either coherent detection or noncoherent detection. For the two cases, we demonstrate the improvement on performance using the proposed weighted despreading sequences and the degradation of performance due to imperfect power control. In the following, it is assumed that the average fading power $\rho$ is perfectly estimated and the $i$ th user's spreading sequence is a random binary sequence with length $N=255$. In this sequence, $\hat{N}_{i}=134, \Gamma_{\{-1,-1,-1\}}^{(i)}=33, \Gamma_{\{-1,-1,1\}}^{(i)}+$ $\Gamma_{\{1,-1,-1\}}^{(i)}=70, \Gamma_{\{-1,1,1\}}^{(i)}+\Gamma_{\{1,1,-1\}}^{(i)}=60, \Gamma_{\{-1,1,-1\}}^{(i)}=$ $36, \Gamma_{\{1,-1,1\}}^{(i)}=31$, and $\Gamma_{\{1,1,1\}}^{(i)}=25$. We use (38) and (55) to compute the probabilities of error for coherent and noncoherent receptions, respectively, at a given set of system parameters.

First, let us consider a RAKE receiver with coherent detection. Fig. 5 shows the BER versus the average signal-to-noise ratio $\bar{\gamma}_{b}$ with diversity order $L_{R}$ varies from one to four. The dashed and solid lines in the figure correspond to the cases of $\gamma=0$ and $\gamma$ tuned to maximize $\hat{H}$ where $\hat{H}$ is the average signal to interference plus noise ratio given in (36). The case of $\gamma=0$ represents the case that the despreading sequence in the receiver consists of rectangular pulses and is identical to the spreading sequence of the reference user. The improvement on performance is obvious when $\gamma$ is tuned to maximize $\hat{H}$. As expected, the BER decreases as the order of diversity $L_{R}$ increases for both cases of $\gamma=0$ and $\gamma$ tuned to maximize $\hat{H}$.

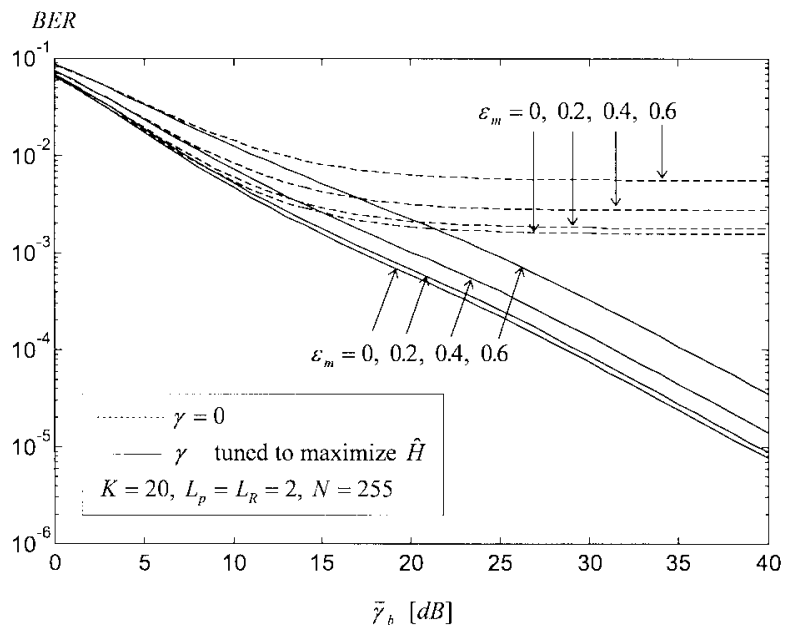

Fig. 6. BER's versus the average signal-to-noise ratio $\bar{\gamma}_{b}$ with the maximum power control error as a parameter for RAKE receiver.

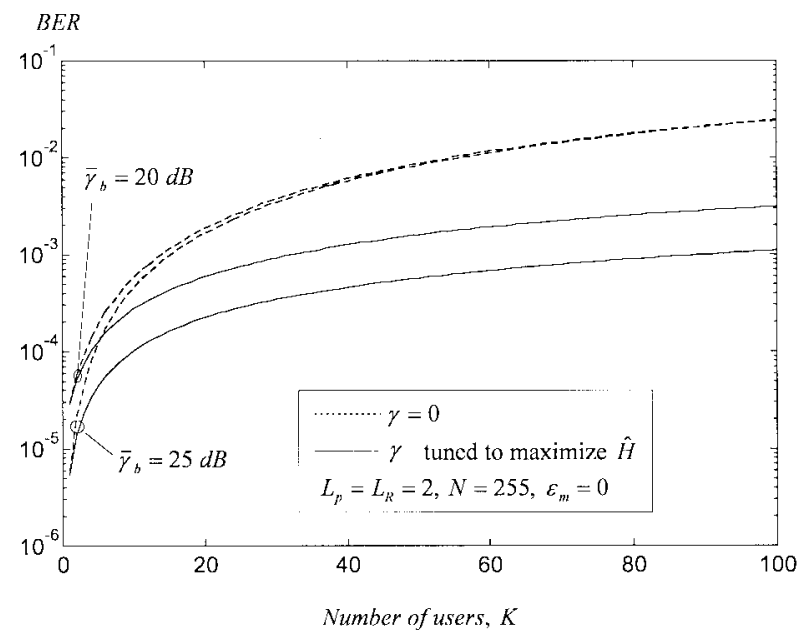

Fig. 7. BER's versus the number of active user $K$ with two values of $\bar{\gamma}_{b}$ for RAKE receiver.

Fig. 6 shows the BER performance versus $\bar{\gamma}_{b}$ with the maximum value of power control error $\varepsilon_{m}$ as a parameter. It is clear that the BER performance degrades for both cases of $\gamma=0$ and $\gamma$ tuned to maximize $\hat{H}$ as $\varepsilon_{m}$ increases. In relative high $\bar{\gamma}_{b}(\geq 15 \mathrm{~dB}), \varepsilon_{m}$ affects the irreducible error probability (or floor of error probability) for the case of $\gamma=0$. As a result, the degradation of system performance can not be compensated by simply increasing $\bar{\gamma}_{b}$. However, for the case of $\gamma$ tuned to maximize $\hat{H}$, the effect on performance due to imperfect power control is equivalent to a reduction of $\bar{\gamma}_{b}$ so that the degradation of system performance can be compensated by increasing $\bar{\gamma}_{b}$. For example, as $\varepsilon_{m}$ increases from 0 to 0.4 , the penalty of $\bar{\gamma}_{b}$ is about $2.5 \mathrm{~dB}$ when $\bar{\gamma}_{b} \geq 10 \mathrm{~dB}$.

In Fig. 7, the BER performance as a function of the number of active users $K$ is plotted for two values of $\bar{\gamma}_{b}$. In the case of $\gamma=0$, the BER for $\bar{\gamma}_{b}=25 \mathrm{~dB}$ is asymptotically close to that for $\bar{\gamma}_{b}=20 \mathrm{~dB}$ as the number of active users $K$ increases. These are situations when the MAI dominates 


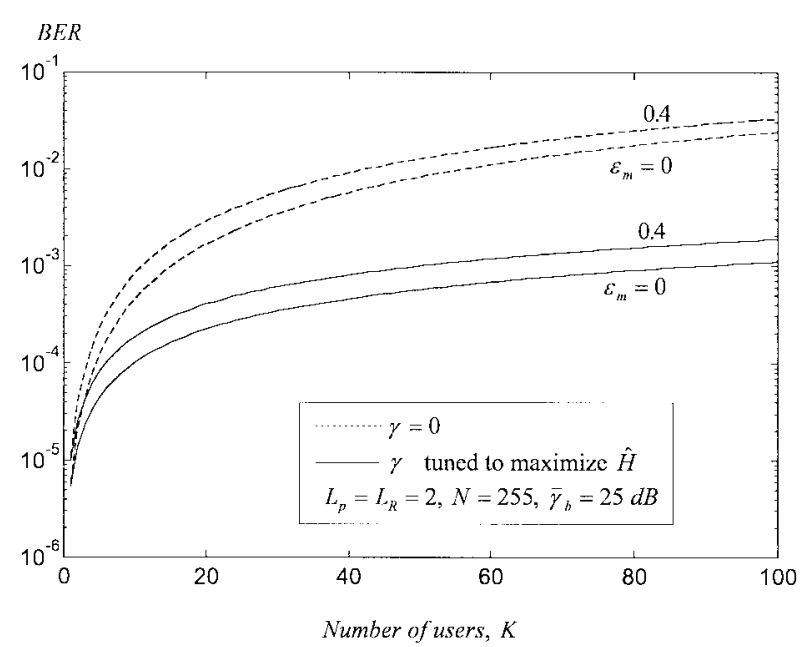

Fig. 8. BER's versus the number of active user $K$ with two values of power control error for RAKE receiver.

over the AWGN in a CDMA system. These graphs reaffirm that the BER performance can not be improved by simply increasing $\bar{\gamma}_{b}$ for the case when the despreading sequences are fixed pulses. In contrast to the rectangular despreading sequence, we can see from this figure that $\bar{\gamma}_{b}$ is a major factor that affects the BER performance for the case of $\gamma$ tuned to maximize $\hat{H}$. For example, when $\bar{\gamma}_{b}$ was increased from 20 to $25 \mathrm{~dB}$, the increase in the number of active users supported at BER $=10^{-3}$ for the cases of $\gamma=0$ and $\gamma$ tuned to maximize $\hat{H}$ are about 1 and 60 , respectively. At BER $=5 \times 10^{-4}$, the increase in the number of active users for the case of $\gamma$ tuned to maximize $\hat{H}$ is about 32 for the same increment of $\bar{\gamma}_{b}$. The graphs indicate that the receiver using weighted despreading sequence outperforms greatly the receiver with fixed or rectangular despreading sequence even a wide range of $\bar{\gamma}_{b}$.

Fig. 8 shows the BER performance as a function of the number of active users $K$ for two values of $\varepsilon_{m}$. When perfect power control is achieved, $\varepsilon_{m}$ is zero. For the case of $\gamma=0$, the number of active users $K$ supported at BER $=10^{-3}$ is about 14 when $\varepsilon_{m}=0$, decreasing to about 11 for the same BER as $\varepsilon_{m}$ increases to 0.4. However, when $\gamma$ is tuned to maximize $\hat{H}$, the number of active users $K$ supported at BER $=10^{-3}$ is about 90 for $\varepsilon_{m}=0$, decreasing to about 48 for the same BER as $\varepsilon_{m}$ increases to 0.4. Although the reduction in the number of active users due to imperfect power control is larger for the case of $\gamma$ tuned to maximize $\hat{H}$ than the case of $\gamma=0$ at a fixed BER performance, the number of active users that can be supported by the latter system is still substantially higher.

Next, we consider a RAKE demodulator with noncoherent detection and DPSK signaling. The curves in Figs. 9-12 correspond to the same cases illustrated in Figs. 5-8, respectively, except the BER's are calculated using (55). In Figs. 9-12, we can see that the RAKE demodulator with noncoherent detection performs slightly poorer than the RAKE receiver with coherent detection at the same operating condition. This is a well-known property. The curves in Figs. 9-12 indicate that the performance of the RAKE demodulator with noncoherent

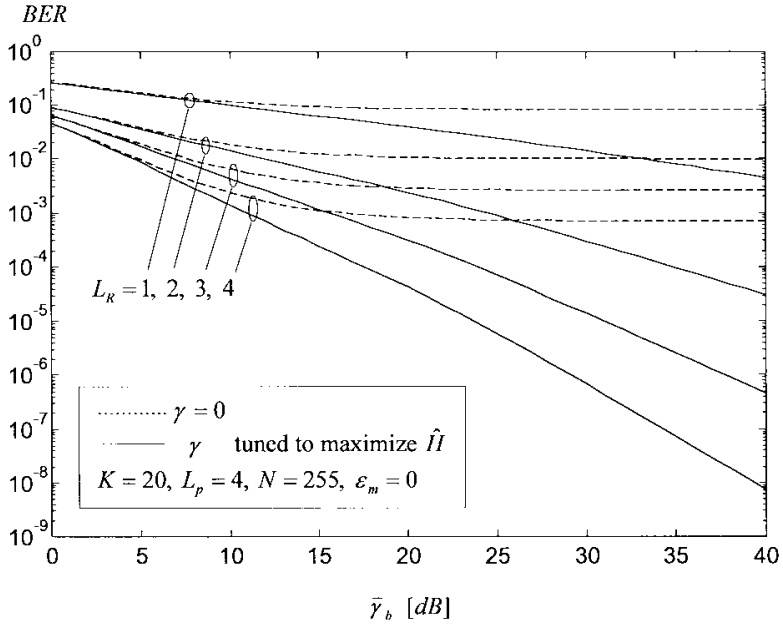

Fig. 9. BER's versus the average signal-to-noise ratio $\bar{\gamma}_{b}$ for RAKE demodulator with different order of diversity.

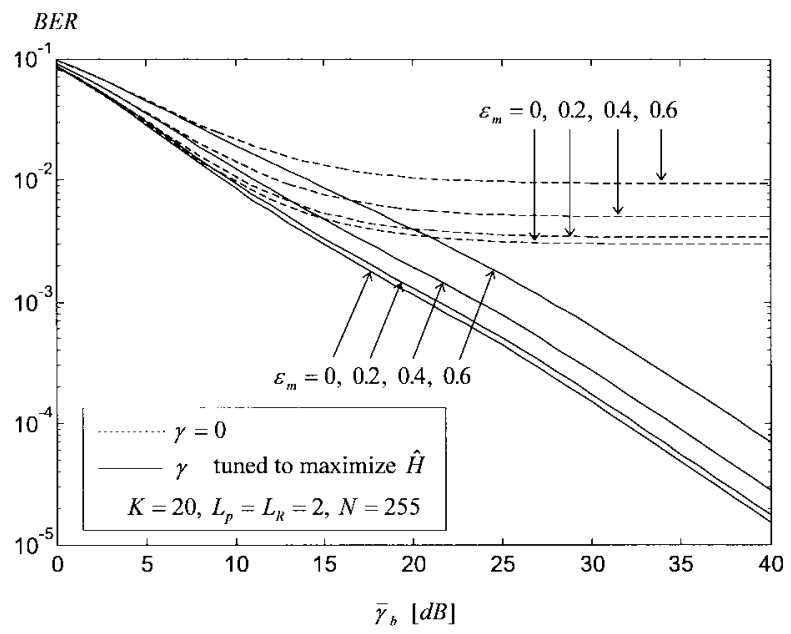

Fig. 10. BER's versus the average signal-to-noise ratio $\bar{\gamma}_{b}$ with the maximum power control error as a parameter for RAKE demodulator.

detection can also be improved tremendously by tuning $\gamma$ to maximize $\hat{H}$. For the case of $\gamma$ tuned to maximize $\hat{H}$, we can increase $\bar{\gamma}_{b}$ for MAI rejection. For example, consider the solid curves shown in Fig. 11, the number of active users supported at BER $=10^{-3}$ increases from 17 to 43 when $\bar{\gamma}_{b}$ is increased from 20 to $25 \mathrm{~dB}$. On the other hand, the increase in the number of active users supported at BER $=10^{-3}$ for the case of $\gamma=0$ is only about 1 for the same increment of $\bar{\gamma}_{b}$.

Fig. 12 shows that the effect of power control error on performance of the RAKE demodulator with noncoherent detection. Clearly, the decrease in the number of active users at a given BER is also larger for the case of $\gamma$ tuned to maximize $\hat{H}$ than the case of $\gamma=0$. From the figure we can see that the penalty of the number of active users supported at BER $=10^{-3}$ for both $\gamma=0$ and $\gamma$ tuned to maximize $\hat{H}$ cases are about 2 and 20, respectively, when $\varepsilon_{m}$ increases from 0 to 0.4 .

For the purpose of comparison, Fig. 13 plots the BER performance of both coherent reception and noncoherent reception for various orders of diversity. We note the difference 


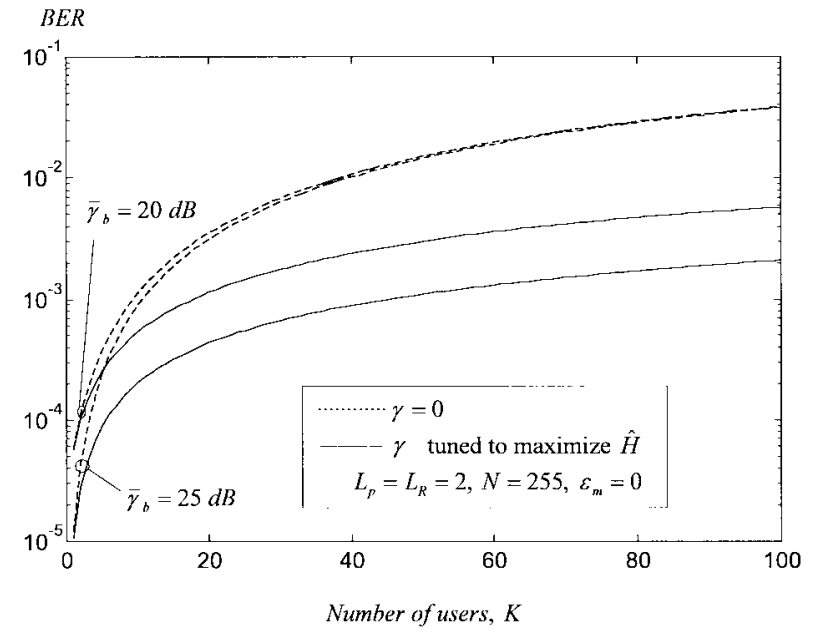

Fig. 11. BER's versus the number of active user $K$ with two values of $\bar{\gamma}_{b}$ for RAKE demodulator.

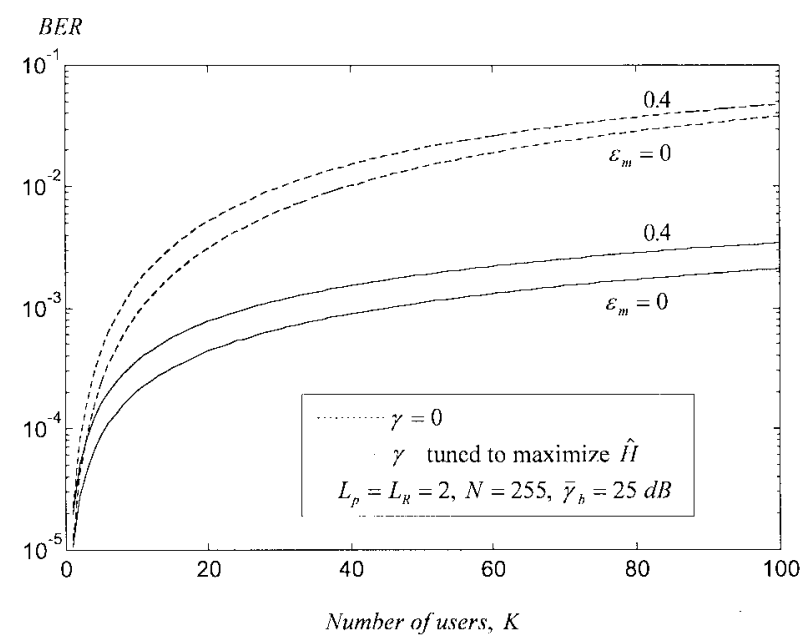

Fig. 12. BER's versus the number of active user $K$ with two values of maximum power control error for RAKE demodulator.

in performance between the two systems is less for low order of diversity. This suggests that the performance gain from diversity is higher for RAKE receiver with coherent detection. From the figure we can also see that when the MAI is insignificant, the BER performance of the two systems for the case of $\gamma$ tuned to maximize $\hat{H}$ is very close to that for the case of $\gamma=0$. For example, when $\bar{\gamma}_{b} \leq 5 \mathrm{~dB}$, the BER's for either system have a negligible difference between both $\gamma=0$ and $\gamma$ tuned to maximize $\hat{H}$ cases. This is because the BER at low average signal-to-noise ratio is mostly caused by AWGN so that tuning $\gamma$ has little effect on the performance.

In Fig. 14, the average signal to interference plus noise ratio $\hat{H}$ is plotted as a function of $\gamma$ for various values of $\bar{\gamma}_{b}$. The solid and dashed curves correspond to the cases of $\varepsilon_{m}=0$ and $\varepsilon_{m}=0.4$, respectively. One can see that the effect on $\hat{H}_{\max }$ due to imperfect power control is not obvious where $\hat{H}_{\max }$ stands for the maximum value of $\hat{H}$ obtained by tuning the parameter $\gamma$. However, as mentioned earlier, the penalty on $\bar{\gamma}_{b}$ at a given BER between $\varepsilon_{m}=0$ and $\varepsilon_{m}=0.4$ is about 2.5 $\mathrm{dB}$ for the case of $\gamma$ tuned to maximize $\hat{H}$ when $\bar{\gamma}_{b} \geq 10 \mathrm{~dB}$.

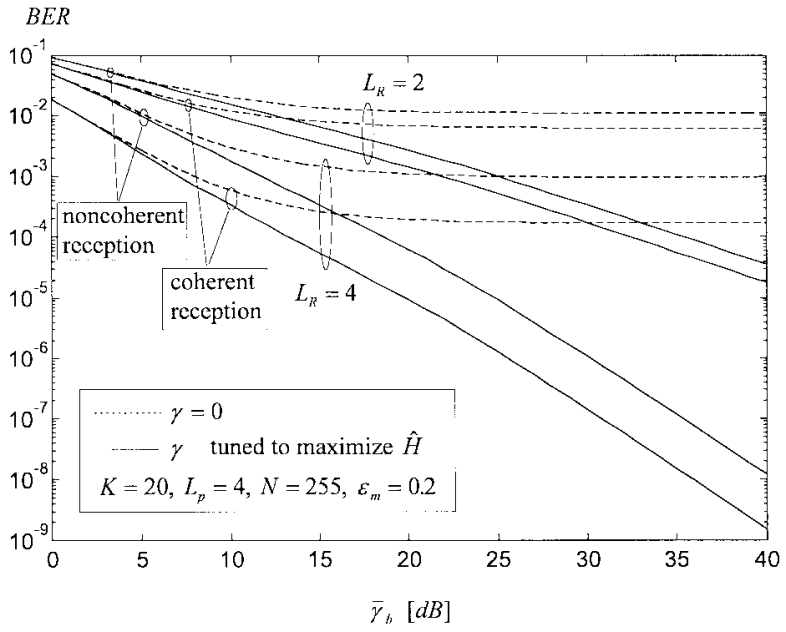

Fig. 13. BER's versus the average signal-to-noise ratio $\bar{\gamma}_{b}$ with two numbers of diversity order for coherent reception (RAKE receiver) and noncoherent reception (RAKE demodulator).

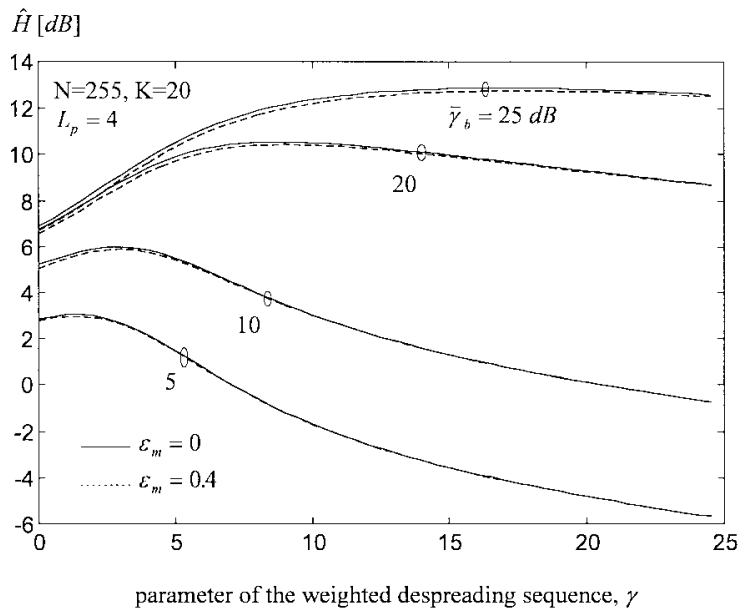

Fig. 14. The average signal to interference plus noise ratio $\hat{H}$ versus the parameter $\gamma$ for various values of the average signal-to-noise ratio $\bar{\gamma}_{b}$ when $\varepsilon_{m}=0$ and $\varepsilon_{m}=0.4$

This suggests that the degree of $\hat{H}_{\max }$ reduction relative to that of BER performance degradation due to imperfect power control is very small. This figure also shows that the difference between $\hat{H}_{\max }$ obtained by tuning $\gamma$ and $\hat{H}$ at $\gamma=0$ is small when $\bar{\gamma}_{b} \leq 5 \mathrm{~dB}$ so that the weighted despreading sequence can be simply replaced by rectangular spreading sequence with little loss of BER performance. On the other hand, when the MAI is significant (e.g., $\overline{\gamma_{b}} \geq 10 \mathrm{~dB}$ ), $\gamma$ should be tuned with respect to each $\bar{\gamma}_{b}$ to maximize $\hat{H}$. This is illustrated by the two top sets of curves in Fig. 14. That is, the gain of $\hat{H}_{\max }$ is much larger than that of $\hat{H}$ at $\gamma=0$ as $\bar{\gamma}_{b}$ increases from 20 to $25 \mathrm{~dB}$.

Finally, we discuss the effects of parameter errors on system performance. First, recall that all numerical results are obtained under the assumption that the average fading power $\rho$ is estimated perfectly. In practice, errors in estimating $\rho$ is unavoidable and hence in obtaining the optimal value of $\gamma$ that maximizes $\hat{H}$. However, it can be seen from Fig. 14 that each curve varies slowly around its maximum point. This implies 
that when the optimal value of $\gamma$ is replaced by its estimated value, the degradation in performance is small. Second, from (36), it is clear that $\hat{H}$ is a function of $\rho$. In order to maximize $\hat{H}, \gamma$ must be tuned explicitly as the channel conditions change. Because $\rho$ represents the average characteristics of the channel and varies slowly, it is reasonable to assume that $\gamma$ can be tuned fast enough to compensate for the change of $\rho$.

\section{CONCLUSIONS}

In this paper, the BER performance of a DS-CDMA system with power control error using RAKE receptions over a multipath Rayleigh fading channel has been evaluated and closed-form solutions for the BER performance have been obtained for both coherent and noncoherent receivers. For MAI rejection, the receivers under consideration employ the despreading sequences weighted by adjustable exponential chip waveforms. Numerical results show that tremendous improvement on performance can be achieved using both multipath diversity and the proposed weighted despreading sequences in the receivers. For both coherent and noncoherent receptions, imperfect power control for the case of $\gamma=0$ increases the irreducible error probability (or floor of error probability) in relative high average signal-to-noise ratio $\bar{\gamma}_{b}$. On the other hand, the penalty on performance with power control error for the case of $\gamma$ tuned to maximize $\hat{H}$ is equivalent to a reduction in the average signal-to-noise ratio at a given BER in a DS-CDMA system. Therefore, when $\gamma$ is tuned to maximize $\hat{H}$, the degradation of performance due to imperfect power control can be compensated by increasing $\bar{\gamma}_{b}$. We also show that the effect due to imperfect power control on the BER performance is significant while that on the maximum value of $\hat{H}$ obtained by tuning $\gamma$ is rather insignificant.

\section{APPENDIX}

In this Appendix, we evaluate the integral

$$
\begin{aligned}
P_{e}^{(i)}= & \frac{1}{2 \varepsilon_{m}} \int_{1-\varepsilon_{m}}^{1+\varepsilon_{m}} P_{e \mid G_{i}}^{(i)} d G_{i} \\
= & \left(\frac{1}{2 \varepsilon_{m}}\right) \sum_{k=0}^{L_{R}-1}\left(\begin{array}{c}
L_{R}-1+k \\
k
\end{array}\right) \int_{1-\varepsilon_{m}}^{1+\varepsilon_{m}} \\
& \cdot\left(\frac{1-\mu}{2}\right)^{L_{R}}\left(\frac{1+\mu}{2}\right)^{k} d G_{i}
\end{aligned}
$$

where $\mu=\sqrt{R_{c} /\left(1+R_{c}\right)}$ and $R_{c}=\hat{H}^{2} G_{i}^{2}$, and we show the result is given by

$$
P_{e}^{(i)}= \begin{cases}\frac{1}{2}-\frac{1}{4 \varepsilon_{m} \hat{H}}\left(\sqrt{a^{2}+1}-\sqrt{b^{2}+1}\right), & L_{R}=1 \\ \bar{\Omega}\left(L_{R}, \varepsilon_{m}, \hat{H}\right), & L_{R} \geq 2\end{cases}
$$

where $a=\left(1+\varepsilon_{m}\right) \hat{H}, b=\left(1-\varepsilon_{m}\right) \hat{H}$, and

$$
\begin{aligned}
\bar{\Omega} & \left(L_{R}, \varepsilon_{m}, \hat{H}\right) \\
= & \sum_{k=0}^{L_{R}-1}\left(\begin{array}{c}
L_{R}-1+k \\
k
\end{array}\right) \sum_{j_{k}=0}^{k}(-1)^{j_{k}}\left(\frac{1}{2}\right)^{L_{R}+j_{k}}\left(\begin{array}{l}
k \\
j_{k}
\end{array}\right) \\
& \cdot\left\{\left[1-\frac{L_{R}+j_{k}}{2 \varepsilon_{m} \hat{H}}\left(\sqrt{a^{2}+1}-\sqrt{b^{2}+1}\right)\right]\right.
\end{aligned}
$$

$$
\begin{aligned}
& \cdot\left(\frac{1}{2 \varepsilon_{m} \hat{H}}\right) U(n-2) \sum_{(n \in \text { even })}^{L_{R}+j_{k}}\left(\begin{array}{c}
L_{R}+j_{k} \\
n
\end{array}\right) \\
& \text {. }\left\{(a-b)-\left(\frac{n}{2}\right) \operatorname{arctg}\left(\frac{a-b}{1+a b}\right)\right. \\
& +U(n-4) \sum_{q=2}^{n / 2}(-1)^{q}\left(\begin{array}{c}
n / 2 \\
n / 2-q
\end{array}\right) \\
& \cdot\left\{\left(\begin{array}{c}
2 q-3 \\
q-1
\end{array}\right)\left(\frac{1}{2^{2 q-3}}\right) \operatorname{arctg}\left(\frac{a-b}{1+a b}\right)\right. \\
& +\left(\frac{1}{2 q-1}\right) \sum_{p=1}^{q-1}\left[\frac{(2 q-1)(2 q-3) \cdots(2 q-2 p+1)}{2^{p}(q-1)(q-2) \cdots(q-p)}\right] \\
& \left.\left.\cdot\left[\frac{a}{\left(a^{2}+1\right)^{q-p}}-\frac{b}{\left(b^{2}+1\right)^{q-p}}\right]\right\}\right\} \\
& -\left(\frac{1}{2 \varepsilon_{m} \hat{H}}\right) U(n-3) \sum_{(n \in \text { odd })}^{L_{R}+j_{k}}\left(\begin{array}{c}
L_{R}+j_{k} \\
n
\end{array}\right) \\
& \cdot\left\{\sum_{i=0}^{(n-1) / 2}(-1)^{i}\left(\begin{array}{c}
(n-1) / 2 \\
(n-1) / 2-i
\end{array}\right)\right. \\
& \text { - } \left.\left.\left[\frac{\left(b^{2}+1\right)^{1 / 2-i}-\left(a^{2}+1\right)^{1 / 2-i}}{2 i-1}\right]\right\}\right\} \text {. }
\end{aligned}
$$

In (A-3), the function $U(j-l)$ for any integers $j$ and $l$ is defined as

$$
U(j-l)= \begin{cases}1, & j \geq l \\ 0, & j<l .\end{cases}
$$

When $L_{R}=2$, and after some algebraic manipulation, (A-3) can be written in the form

$$
\begin{gathered}
\left(\frac{1}{2 \varepsilon_{m}}\right) \int_{1-\varepsilon_{m}}^{1+\varepsilon_{m}} \sum_{k=0}^{L_{R}-1}\left(\begin{array}{c}
L_{R}-1+k \\
k
\end{array}\right) \\
\cdot\left(\frac{1-\mu}{2}\right)^{L_{R}}\left(\frac{1+\mu}{2}\right)^{k} d G_{i} \\
=\frac{1}{2}-\frac{1}{4 \varepsilon_{m} \hat{H}}\left(\sqrt{a^{2}+1}-\sqrt{b^{2}+1}\right)-\sum_{d=2}^{L_{R}} \\
\cdot \frac{\left(\begin{array}{c}
2 d-2 \\
d-1
\end{array}\right)}{2^{2 d} \varepsilon_{m} \hat{H}(2 d-3)}\left\{\left(b^{2}+1\right)^{(3-2 d) / 2}\right. \\
\left.-\left(a^{2}+1\right)^{(3-2 d) / 2}\right\} .
\end{gathered}
$$

We proceed by using the method of induction. Assuming that (A-5) is true for an arbitrary positive integer $L_{R}$, we prove that (A-5) is also true when $L_{R}+1$ replaces $L_{R}$ in this equation. By writing $L_{R}+1$ in place of $L_{R}$ in (A-1) and noting that $\bar{\Omega}\left(L_{R}, \varepsilon_{m}, \hat{H}\right)=P_{e}^{(i)}$ for $L_{R} 2$, we obtain

$$
\begin{aligned}
\bar{\Omega}\left(\hat{L}_{R}, \varepsilon_{m}, \hat{H}\right) & \\
= & \left(\frac{1}{2 \varepsilon_{m}}\right) \int_{1-\varepsilon_{m}}^{1+\varepsilon_{m}} \sum_{k=0}^{\hat{L}_{R}-1}\left(\begin{array}{c}
\hat{L}_{R}-1+k \\
k
\end{array}\right) \\
& \cdot\left(\frac{1-\mu}{2}\right)^{\hat{L}_{R}}\left(\frac{1+\mu}{2}\right)^{k} d G_{i}
\end{aligned}
$$


where $\hat{L}_{R}=L_{R}+1$. The RHS of (A-6) is the same as the left-hand side (LHS) of (A-5), except that $\hat{L}_{R}$ replaces $L_{R}$. Thus, the previous expression may be expressed in the form

$$
\begin{gathered}
\bar{\Omega}\left(\hat{L}_{R}, \varepsilon_{m}, \hat{H}\right) \\
=\frac{1}{2}-\frac{1}{4 \varepsilon_{m} \hat{H}}\left(\sqrt{a^{2}+1}-\sqrt{b^{2}+1}\right)-\sum_{d=2}^{\hat{L}_{R}} \\
\cdot \frac{\left(\begin{array}{c}
2 d-2 \\
d-1
\end{array}\right)}{2^{2 d} \varepsilon_{m} \hat{H}(2 d-3)}\left\{\left(b^{2}+1\right)^{(3-2 d) / 2}\right. \\
\left.-\left(a^{2}+1\right)^{(3-2 d) / 2}\right\}
\end{gathered}
$$

which is of the same form as the RHS of (A-5), except that $\hat{L}_{R}$ replaces $L_{R}$. Hence, if (A-5) is true for the index $L_{R}$, it is true for $\hat{L}_{R}\left(=L_{R}+1\right)$, which completes the prove.

\section{REFERENCES}

[1] W. C. Y. Lee, "Overview of cellular CDMA," IEEE Trans. Veh. Technol., vol. 40, pp. 291-302, May 1991.

[2] K. S. Gilhousen et al., "On the capacity of a cellular CDMA system," IEEE Trans. Veh. Technol., vol. 40, pp. 303-312, May 1991.

[3] R. L. Pichholtz, L. B. Milstein, and D. L. Schilling, "Spread spectrum for mobile communications," IEEE Trans. Veh. Technol., vol. 40, pp. 313-322, May 1991

[4] M. B. Pursley, "Performance evaluation for phase-coded spreadspectrum multiple-access communication-Part I: System analysis," IEEE Trans. Commun., vol. COM-25, pp. 795-799, Aug. 1977.

[5] K. Yao, "Error probability of asynchronous spread spectrum multiple access communication systems," IEEE Trans. Commun., vol. COM-25, pp. 803-809, Aug. 1977 .

[6] E. A. Geraniotis and M. B. Pursley, "Performance of coherent direct sequence spread-spectrum communications over specular multipath fading channels," IEEE Trans. Commun., vol. COM-33, pp. 502-508, June 1985.

[7] H. Xiang, "Binary code-division multiple-access systems operating in multipath fading, noise channels," IEEE Trans. Commun., vol. COM-33, pp. 775-784, Aug. 1985 .

[8] G. L. Turin, "The effects of multipath and fading on the performance of direct-sequence CDMA systems," IEEE J. Select. Areas Commun., vol. COM-35, pp. 1189-1198, Nov. 1987.

[9] J. S. Lehnert and M. B. Pursley, "Error probability for binary direct sequence spread-spectrum communications with random signature sequences," IEEE Trans. Commun., vol. COM-35, pp. 87-98, Jan. 1987

[10] M. Kavehrad and B. Ramamurthi, "Direct-sequence spread spectrum with DPSK modulation and diversity for indoor wireless communications," IEEE Trans. Commun., vol. COM-35, pp. 224-236, Feb. 1987.

[11] J. Wang, M. Moeneclaey, and L. B. Milstein, "DS-CDMA with predetection diversity for indoor radio communications," IEEE Trans. Commun., vol. 42, pp. 1929-1938, Feb./Mar./Apr. 1994

[12] T. Eng and L. B. Milstein, "Comparison of hybrid FDMA/CDMA systems in frequency selective Rayleigh fading," IEEE J. Select. Areas Commun., vol. 12, pp. 938-951, June 1994.

[13] N. M. Blachman and S. H. Mousavinezhad, "The spectrum of the square of a synchronous random pulse train," IEEE Trans. Commun., vol. 38 , pp. 13-17, Jan. 1990.

[14] A. D. Whalen, Detection of Signals in Noise. New York: Academic, 1971.

[15] A. M. Monk, M. Davis, L. B. Milstein, and C. H. Helstrom, "A noisewhitening approach to multiple access noise rejection-Part I: Theory and background," IEEE J. Select. Areas Commun., vol. 12, pp. 817-827, June 1994.

[16] B. R. Vojcic, R. L. Pickholtz, and L. B. Milstein, "Performance of DSCDMA with imperfect power control operating over a low earth orbiting satellite link," IEEE J. Select. Areas Commun., vol. 12, pp. 560-567, May 1994.

[17] Y. Huang and T. S. Ng, "Performance of coherent receiver with weighted despreading sequence for DS-CDMA," Electron. Lett., vol. 33, pp. 23-25, Jan. 1997.

[18] _ "A DS-CDMA system using despreading sequences weighted by adjustable chip waveforms," to be published.
[19] D. L. Noneaker and M. B. Pursley, "The effects of spreading sequence selection on DS spread spectrum communications with selective fading and two forms of rake reception," in Proc. 1992 Global Telecommun. Conf., Dec. 1992, vol. 4, pp. 66-70.

[20] G. L. Stüber and C. Kchao, "Analysis of a multiple-cell direct-sequence CDMA cellular mobile radio system," IEEE J. Select. Areas Commun. vol. 10, pp. 669-679, May 1992.

[21] J. G. Proakis, Digital Communications. New York: McGraw-Hill, 1989.

[22] J. H. Park, Jr., "On binary DPSK detection," IEEE Trans. Commun., vol. COM-26, pp. 484-486, Apr. 1978

[23] T. Eng and L. B. Milstein, "Coherent DS-CDMA performance in Nakagami multipath fading," IEEE Trans. Commun., vol. 43, pp. 1134-1143, Feb./Mar./Apr. 1995

[24] G. L. Turin et al., "A statistical model for urban radio propagation," IEEE Trans. Veh. Technol., vol. VT-28, pp. 213-224, Aug. 1979.

[25] A. A. M. Saleh and R. A. Valenzuela, "A statistical model for indoor multipath propagation," IEEE J. Select. Areas Commun., vol. SAC-5, pp. 128-137, Feb. 1987

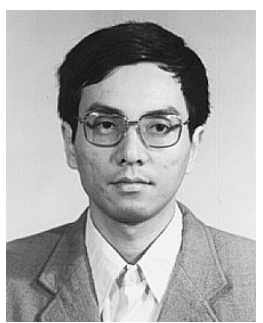

Yuejin Huang (S'95-M'98) received the B.Sc. degree from Sichuan University, China, in 1982, the M.Sc. degree from the Optics and Electronics Institute, Chinese Academy of Sciences, China, in 1988, and the Ph.D. degree from the University of Hong Kong, Hong Kong, in 1998, all in electrical engineering.

From 1982 to 1985, he was an Electrical Engineer at the Southwest Physics Institute of Chinese National Nuclear Corporation. From 1988 to 1994, he was a Member of the Technical Staff at the Nanjing Astronomical Instruments Research Center, Chinese Academy of Sciences, where his duties were the analysis and design of control systems. During his Ph.D. studies (1994-1998), he was a Teaching and Research Assistant in the Department of Electrical and Electronic Engineering, University of Hong Kong, where he researched spread-spectrum communications. Currently, he is with McGill University, Montreal, P.Q., Canada, where he is a Post-Doctoral Fellow in wireless communications systems. His research interests include spread-spectrum communications, signal processing, automatic control, and microprocessor-based instruments.

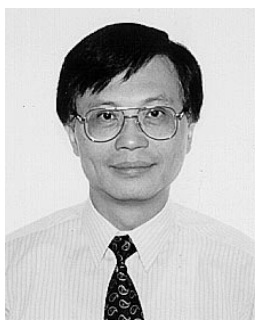

T. S. Ng (S'74-M'78-SM'90) received the B.Sc (Eng.) degree from the University of Hong Kong, Hong Kong, in 1972 and the M.Eng.Sc. and Ph.D degrees from the University of Newcastle, Australia, in 1974 and 1977, respectively, all in electrical engineering.

He was an Electrical Engineer at BHP Steel International, Australia, from 1972 to 1974. From 1977 to 1990 , he was a Lecturer, Senior Lecturer, and Reader at the University of Wollongong, Australia. In 1991, he was appointed Professor and Chair of Electronic Engineering at the University of Hong Kong, a position he currently holds. His current research interests include spread-spectrum techniques, digital signal processing, mobile communication systems, and engineering applications of artificial intelligence. He has published more than 150 international journal and conference papers. He is currently a Regional Editor of Engineering Applications of Artificial Intelligence.

Dr. Ng served the IEEE Hong Kong Section as the Honorary Treasurer (1995 and 1996) and the Technical Program Coordinator (1993 and 1994). He also served the IEE Hong Kong as the Chairman (1996-1997), Vice Chairman (1995-1996), and Conference Coordinator (1993-1995). He has served in different capacities in many international conferences, in particular, he was the General Cochair of the IEEE ISCAS'97. He served on the Hong Kong Government Industry Technology Development Council Electronics Committee (1991-1996) and is currently a member of the Engineering Panel and the Committee for Cooperative Research Centers of the Hong Kong Research Grants Council. He was awarded the Honorary Doctor of Engineering Degree by the University of Newcastle, Australia, in August 1997 for his services in Hong Kong to higher education generally and to engineering education specifically. 